\title{
Kinetic energy budget of the largest scales in turbulent pipe flow
}

\author{
C. Bauer, ${ }^{1,2, *}$ A. von Kameke, ${ }^{3}$ and C. Wagner ${ }^{1,2}$ \\ ${ }^{1}$ Institute of Aerodynamics and Flow Technology, German Aerospace Center, \\ Bunsenstraße 10, 37073 Göttingen, Germany \\ ${ }^{2}$ Institute of Thermodynamics and Fluid Mechanics, Technische Universität Ilmenau, \\ Helmholtzring 1, 98693 Ilmenau, Germany \\ ${ }^{3}$ Institute of Multiphase Flows, Hamburg University of Technology, \\ Eißendorfer Straße 38, 21073 Hamburg, Germany
}

(Received 14 November 2018; published 10 June 2019)

\begin{abstract}
So-called very-large-scale motions (VLSM) have been observed in turbulent pipe flows recently. It was discovered that they carry a substantial fraction of turbulent kinetic energy. However, the question how they gain and loose their energy from other scales has not been rigorously studied yet. Hence, the present study is intended to investigate how energy is transferred toward and away from the very-large scales. The inter- and intrascale energy flux in turbulent pipe flow is analyzed by means of the $\left\langle\overline{u_{z}^{\prime}} \overline{u_{z}^{\prime}}\right\rangle$-budget equation of the two-dimensionally filtered streamwise fluctuating velocity field $\overline{u_{z}^{\prime}}$ obtained from a direct numerical simulation at $\operatorname{Re}_{\tau}=1500$. We show that the largest scales of motion gain their energy in the logarithmic layer through the production term of the low-pass filtered budget equation. In contrast to the small-scale energy transfer near the wall, no mean backscattering of energy is observed toward VLSM. Instantaneous flow field realizations as well as conditional averages, on the contrary, show backscattering into negative ejecting VLSM up to $y^{+}=200$, which is overcompensated by even stronger forward scattering from positive sweeping VLSM. This behavior opposes the small-scale energy transfer near the wall, where backscattering is associated with high-speed sweeping motions.
\end{abstract}

DOI: 10.1103/PhysRevFluids.4.064607

\section{INTRODUCTION}

In recent years very-large-scale motions (VLSM) have become an increasingly important topic in the analysis of wall-bounded turbulence. They were first observed by Kim and Adrian [1] as a large wavelength peak in the pre-multiplied energy spectrum of the streamwise fluctuations in turbulent pipe flow. These structures become more energetic with increasing Reynolds numbers [2]. Although they are found in the outer flow, they penetrate deep into the buffer layer and interact with the near-wall cycle, thus influencing turbulence statistics very close to the wall $[3,4]$. VLSM carry a substantial amount of turbulent kinetic energy and can be characterized by high-momentum sweeps and low-momentum ejections similar to the small scales in the vicinity of the wall [5]. The reason why VLSM become energetic and the question how energy is distributed away from them needs further clarification. Earlier studies aiming at the interscale energy flux in wall-bounded turbulent flows revealed an inverse energy cascade - also referred

*christian.bauer@dlr.de 
to as backscatter events - contradicting the classical theory of the turbulent cascade as an energy transfer from larger scales to smaller ones only $[6,7]$. Such an inverse cascade is traditionally observed in two-dimensional turbulent flows [8] or in the case of rotating turbulence [9]. However, for wall-bounded turbulence Härtel et al. [6] found that both strong forward- and backscatter events are related to strong shear layers - large negative correlations of the stream- and wall-normal velocity fluctuations $u^{\prime} v^{\prime}$. With the aid of conditional averaging Piomelli et al. [7] revealed that forward scatter events are rather related to ejections $\left(u^{\prime}<0, v^{\prime}>0\right)$, while the transfer of energy from subgrid to grid scales is mainly attributed to motions sweeping toward the solid boundary $\left(u^{\prime}>0, v^{\prime}<0\right)$. These early investigations of the interscale energy flux using fully resolved direct numerical simulations (DNS) of turbulent plane channel and pipe flow were restricted to Reynolds numbers as low as $\operatorname{Re}_{\tau}=180$ and computational domains with a streamwise extent not larger than $L_{x}<4 \pi h$, where $h$ is the channel half height. Due to their low Reynolds number and short computational domain, they were not able to observe and analyze the largest scale of motions which are the subject of our study. Albeit recent studies by Cimarelli et al. [10-15] provide important advances in this regard by taking advantage of the space-scale duality of the generalized Kolmogorov equation (GKE). Cimarelli et al. [15] have revealed two different mechanisms of interscale energy transfer toward large scales away from the wall and determined the locations of the interscale energy sources responsible for this redistribution of energy. By untangling the complex picture of field lines of the combined phase-space of the spatial and so-called scale energy fluxes, they found an attached and a detached cascade. In both cascades backward scale energy fluxes play a central role and feed larger scales away from the wall. A drawback of this method is that no direct structural information of the filtered velocity fields at the scale of interest is derived, which makes it difficult to characterize the type of structures involved in the processes. Cho et al. [16] and Lee and Moser [17] studied the budget equations in turbulent channel flow by means of a spectral analysis for Reynolds numbers of $\operatorname{Re}_{\tau}=1700$ and $\operatorname{Re}_{\tau}=5200$, respectively. The former study focused on spanwise scales only. Hence, they did not explicitly distinguish between LSM and VLSM since these correspond to the same spanwise wavelengths. They found turbulent production at spanwise scales which are proportional to the wall distance in the log-layer, whereas the related spanwise wavelength of the dissipation spectra is proportional to the Kolmogorov length scale. Although they confirmed - via the visualization of triadic interactions - that the classical energy cascade is the dominant transfer mechanism, they also found an inverse energy cascade in both the streamwise and spanwise velocity component from small to large scales. The latter study by Lee and Moser [17] focused on two-dimensional spectra of the budget equation terms. As they also took the streamwise wave modes into account, they were able to observe turbulent production at scales as large as VLSM, particularly away from the wall. Regarding the spanwise scales, they acknowledged the findings of Cho et al. [16] that production occurs at spanwise scales increasing with the distance from the wall.

The aim of this work is to analyze the kinetic energy budget of the filtered velocity field similar to the previous analysis of two-dimensional turbulence [8]. In this way, we intend to shed light on the relevant fluxes and sources that sustain the largest scale of motions in turbulent pipe flow. The characterization of the structural information on the largest scales of the velocity field is also part of this study. This is done using a DNS of $\mathrm{Re}_{\tau}=1500$ in periodic computational domains of $L=42 R$, where $R$ is the pipe radius. Such a large computational domain is required to fully capture the largest motions in turbulent wall-bounded flow in numerical simulations, as previous studies have already shown [18-20]. Since energetic wavelengths related to VLSM appear in the streamwise velocity component only, the streamwise Reynolds stress budget equation of a filtered velocity field is analyzed. The budget terms are examined by means of one- and two-point turbulence statistics as well as by instantaneous flow-field realizations. Moreover, the high-speed (sweeping) and low-speed (ejecting) VLSM are related to the budget terms by taking advantage of quadrantwise conditionally averaged statistics. 
TABLE I. Turbulent pipe-flow simulation case. $L$ is the pipe length. $N_{z}, N_{\varphi}$, and $N_{r}$ are the number of grid points with respect to the axial, azimuthal, and radial direction, respectively. $\Delta z^{+}$, axial grid spacing; $R^{+} \Delta \varphi$, azimuthal grid spacing at the wall; $\Delta r_{\min }^{+}$and $\Delta r_{\max }^{+}$, minimal and maximal radial grid spacing, respectively. All grid spacings normalized by wall units. $N_{t}$ is number of instantaneous flow field realizations used to compute statistical averages. $\Delta t^{+}=\Delta t u_{\tau}^{2} / \nu$ is the time interval between two subsequent samples in viscous units and $\Delta t_{b}=\Delta t u_{b} / R$ is the interval in bulk units.

\begin{tabular}{cccccccccc}
\hline \hline $\mathrm{Re}_{\tau}$ & $L / R$ & $N_{z} \times N_{\varphi} \times N r$ & $\Delta z^{+}$ & $R^{+} \Delta \varphi$ & $\Delta r_{\min }^{+}$ & $\Delta r_{\max }^{+}$ & $N_{t}$ & $\Delta t^{+}$ & $\Delta t_{b}$ \\
\hline 1500 & 42 & $8192 \times 2048 \times 408$ & 7.7 & 4.6 & 0.49 & 7.8 & 50 & 480 & 6.4 \\
\hline \hline
\end{tabular}

\section{NUMERICAL METHODOLOGY}

\section{A. Assessment of the data set}

The analysis of the present work is based on a data set obtained from a direct numerical simulation of turbulent pipe flow used by Bauer et al. [4]. They applied a fourth-order finite-volume method [21] to solve the incompressible Navier-Stokes equation in the dimensionless form

$$
\begin{gathered}
\frac{\partial u_{i}}{\partial t}+u_{j} \frac{\partial u_{i}}{\partial x_{j}}=-\frac{\partial p}{\partial x_{i}}+\frac{1}{\operatorname{Re}_{\tau}} \frac{\partial^{2} u_{i}}{\partial x_{j}^{2}}, \quad i=1,2,3, \\
\frac{\partial u_{i}}{\partial x_{i}}=0,
\end{gathered}
$$

where $\operatorname{Re}_{\tau}=u_{\tau} R / v$ is the friction Reynolds number, based on friction velocity, pipe radius and kinematic viscosity. The flow geometry, an annular smooth pipe with length $L$ and radius $R$, is discretized via staggered grids in a cylindrical coordinate system, where $z$ is the axial, $\varphi$ the azimuthal, and $r$ the radial coordinate. The wall-normal coordinate pointing toward the pipe axis is hereinafter denoted as $y=(R-r)$ and in wall units $y^{+}=y / \delta_{v}$, with $\delta_{v}=v / u_{\tau}$ being the viscous length scale. Henceforth, the + superscript refers to quantities normalized in viscous units. Table I shows the numerical set-up used within this study. Angled brackets denote averaged variables

$$
\langle u\rangle(r)=\frac{1}{L} \frac{1}{2 \pi r} \frac{1}{\Delta T} \int_{t=t_{0}}^{t_{0}+\Delta T} \int_{z=0}^{L} \int_{\varphi=0}^{2 \pi} u(z, \varphi, r, t) r d \varphi d z d t
$$

where the average is computed in space and time by taking advantage of the flow homogeneity in the stream- and spanwise direction. The temporal average is obtained from 50 instantaneous flow field realizations with an temporal increment of $\Delta t^{+}=480$ in viscous units. In the following, the prime superscript is used to denote the velocity fluctuation of a component with respect to its mean $\left(u_{i}^{\prime}=u_{i}-\left\langle u_{i}\right\rangle\right)$

\section{B. Spatial filtering}

Analyzing the energy transfer between different turbulent scales involves spatial filtering. We use a sharp spectral cutoff filter with respect to the two homogeneous directions $(z, \varphi)$. No filter is applied in the radial direction $(r)$ to avoid complications arising due to the inhomogeneity. Since the most energetic turbulent coherent structures - small scales as well as large scales are highly anisotropic, anisotropic spatial filtering is applied. These structures are much longer than they are wide, and the application of isotropic filtering would, thus, cut off all spanwise modes of one type of motions, while several streamwise modes associated with the same type of motions would not be cut off. To divide the range of scales between small-scale motions, on the one hand, and large- and very-large-scale motions, on the other hand, one usually applies a one-dimensional filter in spanwise direction [16,22]. To further separate VLSM from all smaller motions, we apply two-dimensional filtering with respect to both the stream- and spanwise direction, 
(a)

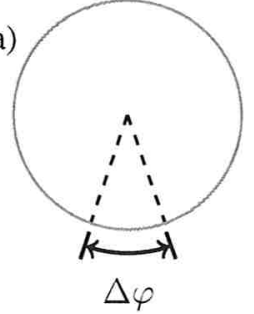

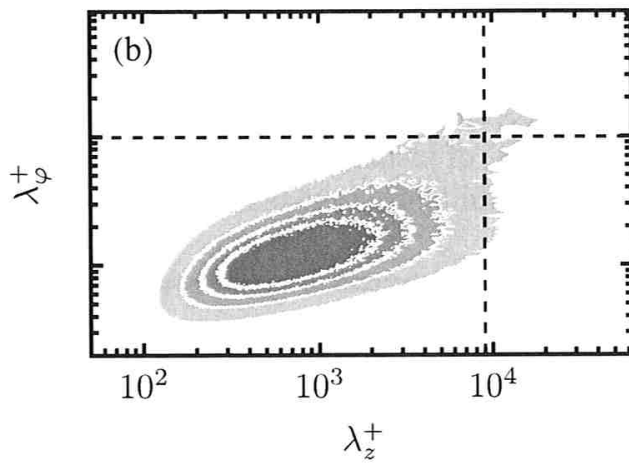

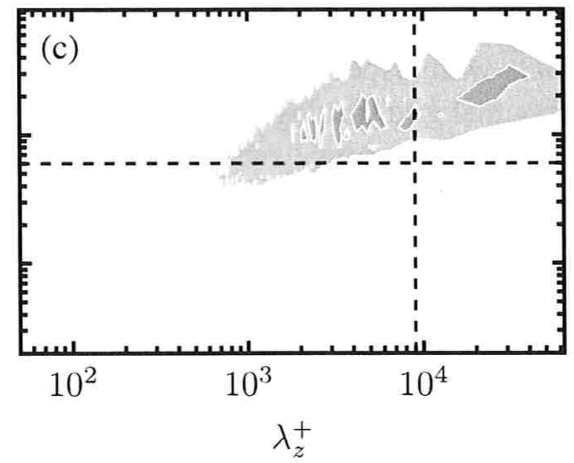

FIG. 1. (a) Spanwise filter in the pipe cross section. (b), (c) Two-dimensional premultiplied energy spectra of the streamwise velocity component $\kappa_{z} \kappa_{\varphi} \phi_{z z}$ as functions of the streamwise wavelength $\lambda_{z}$ and the spanwise wavelength $\lambda_{\varphi}$ at $y^{+}=15$ (b) and $y / R=0.4$ (c). All units are normalized by wall units. $\kappa=\pi / \lambda$. Isosurfaces denote values ranging from 0.12 (light red) to 0.48 (dark red) with an increment of 0.12 .

having a larger filter length in streamwise than in spanwise direction. While the streamwise filter length $\lambda_{z}$ is fixed in spatial units, the spanwise filter length $\Delta \varphi$ is kept constant in radian measure, as shown in Fig. 1(a). Since the considered turbulent scales are most energetic near the wall small scales in the buffer layer and LSM as well as VLSM in the logarithmic layer - in the following, we use the maximum spatial filter length in the spanwise direction at $r=R$ in wall units, namely $\lambda_{\varphi}^{+}=R^{+} \Delta \varphi$, for comparison. The filter pays tribute to the flow geometry, where the scale space in the spanwise direction becomes smaller in spatial units toward the cylindrical axis. Hence, the relevance of the energy budget is limited near the cylindrical axis, where the spanwise filter length is small in spatial units. However, wall-bounded turbulence is least energetic in this region of the flow and small-scale motions do not exist here. Throughout this study, two different filter configurations are used. The first, in the following referred to as configuration A, where filter lengths read $\lambda_{z}^{+}=76, \lambda_{\varphi}^{+}=R^{+} \Delta \varphi=42$, matches the one used in Härtel et al. [6]. A comparison between the TKE interscale energy flux computed from the current data set and the one computed by Härtel et al. [6] is depicted in Fig. 14 in the Appendices. These filter lengths are of the same order of magnitude as classical large-eddy simulation (LES) grid resolutions. The filtered structures are therefore comparable to the subgrid-scale structures in an LES. Configuration A is used to reveal effects attributed to viscous small-scale motions. The second filter configuration (B) is used to uncover the largest scales of turbulent motion, which are less energetic than the small-scale motions associated with the near-wall cycle. To determine the filter lengths in a way that they efficiently divide the range of scales into a small-scale and LSM part, on one hand, and a VLSM part, on the other hand, we use two-dimensional premultiplied energy spectra, as shown in Fig. 1, at two prominent wall-normal locations from the wall $\left[y^{+}=15\right.$, Fig. 1(b); $y / R=0.4$, Fig. 1(c) $]$. Filter lengths of $\lambda_{z}^{+}=9000$ and $\Delta \varphi=2 / 3\left(\lambda_{\varphi}^{+}=1000\right)$, indicated by dashed lines in Fig. 1, meet this requirement and are thus chosen for further study (filter configuration B). Although VLSM become increasingly energetic with the Reynolds number [23], Fig. 1 also corroborates that a Reynolds number as low as $\operatorname{Re}_{\tau}=1500$ exhibits sufficient scale separation for the analysis of the interaction between small turbulent scales and VLSM. Moreover, the variation of the spanwise filter length in wall units does not affect the results significantly in the region of interest, since energetic turbulent scales are sufficiently separated [Figs. 1(b) and 1(c)].

\section{The streamwise Reynolds stress budget equation of the filtered velocity field}

To analyze the streamwise kinetic energy contribution of large scales only, the filtering operation,

$$
\bar{u}_{i}(\vec{x}, t)=\int G(\vec{r}, \vec{x}) u_{i}(\vec{x}-\vec{r}, t) d \vec{r},
$$


is applied to Eq. (1). Here $G$ denotes a sharp spectral cut-off filter defined as

$$
G(\kappa)=\left\{\begin{array}{ll}
0 & \text { if } \kappa \leqslant \kappa_{c} \\
1 & \text { if } \kappa>\kappa_{c}
\end{array},\right.
$$

in spectral space with a cutoff wave number $\kappa_{c}=\pi / \lambda$. After subtraction of the Reynolds equation and multiplication with $\overline{u_{i}^{\prime}}$, the Reynolds stress budget equation of the filtered velocity field is obtained. For fully developed turbulent pipe flow the budget equation for the streamwise component is

$$
\begin{aligned}
0= & -\frac{1}{r} \frac{d\left(r\left\langle\overline{u_{r}^{\prime}} \cdot \overline{u_{z}^{\prime}} \cdot \overline{u_{z}^{\prime}}\right\rangle\right)}{d r}+\frac{1}{\operatorname{Re}_{\tau}} \frac{1}{r} \frac{d}{d r}\left(r \frac{d\left\langle\overline{u_{z}^{\prime}} \cdot \overline{u_{z}^{\prime}}\right\rangle}{d r}\right)+2\left\langle\overline{p^{\prime}} \frac{\partial \overline{u_{z}^{\prime}}}{\partial z}\right\rangle-2\left\langle\overline{u_{z}^{\prime}} \cdot \overline{u_{r}^{\prime}}\right\rangle \frac{d\left\langle u_{z}\right\rangle}{d r} \\
& -\frac{2}{\operatorname{Re}_{\tau}}\left(\left\langle\left(\frac{\partial \overline{u_{z}^{\prime}}}{\partial z}\right)^{2}\right\rangle+\left\langle\frac{1}{r^{2}}\left(\frac{\partial \overline{u_{z}^{\prime}}}{\partial \varphi}\right)^{2}\right\rangle+\left\langle\left(\frac{\partial \overline{u_{z}^{\prime}}}{\partial r}\right)^{2}\right\rangle\right) \\
& -\frac{2}{r} \frac{d\left(r\left(\overline{u_{z}^{\prime}} \tau_{r z}^{R}\right\rangle\right)}{d r}+2\left(\left\langle\tau_{z z}^{R} \frac{\partial \overline{u_{z}^{\prime}}}{\partial z}\right\rangle+\left\langle\tau_{\varphi z}^{R} \frac{1}{r} \frac{\partial \overline{u_{z}^{\prime}}}{\partial \varphi}\right\rangle+\left\langle\tau_{r z}^{R} \frac{\partial \overline{u_{z}^{\prime}}}{\partial r}\right\rangle\right),
\end{aligned}
$$

where $\tau_{i j}^{R}=\overline{u_{i}^{\prime} u_{j}^{\prime}}-\overline{u_{i}^{\prime}} \bar{u}_{j}^{\prime}$ are the residual stresses. The terms in Eq. (6) read from left to right are denoted as the turbulent transport term (TD), the viscous diffusion (VD) term, the pressure-strain term (PS), the production term (P), the dissipation term (D), the subfilter-scale diffusion term (SD), and the interscale energy flux (EF) of the filtered streamwise velocity. Within the given framework, the terms TD, VD, PS and SD describe the intra-scale energy transport, i.e., the transport of energy of scales larger than the filter length in physical space. The production term in Eq. (6) reflects the amount of streamwise turbulent kinetic energy transferred from the mean field into turbulent coherent structures, which are larger than the filter length. The dissipation term in Eq. (6), on the contrary, depicts the amount of energy directly dissipated from scales larger than the filter length. Finally, the interscale energy flux term describes the amount of energy transferred from scales smaller than the filter length into larger ones - or vice versa - and, hence, acts as a source or sink - in Eq. (6).

\section{STREAMWISE TURBULENCE INTENSITY BUDGET OF THE LARGEST SCALES}

The streamwise velocity component contributes most to the turbulent kinetic energy in wallbounded turbulent flows [24]. Figure 2 shows the turbulent intensities for the full as well as for the filtered velocity field. In particular, it demonstrates that the amount of turbulent kinetic energy carried by VLSM is dominated by the streamwise component (dashed black line). This justifies that the present study only focusses on the streamwise Reynolds stress budget equation of the large-scale filtered field [Eq. (6)]. While the streamwise turbulence intensity peak of the unfiltered field and the small-scale filtered field is located at a wall distance of $y^{+} \approx 15$, where wall-layer streaks are the dominant turbulent species, the streamwise turbulence intensity peak of the large-scale filtered field is found in the logarithmic layer at $y^{+} \approx 390$ or $y=0.26 R$ in bulk units, respectively. The single budget terms of Eq. (6) are depicted in Fig. 3, where the focus is on the near-wall region [Figs. 3(a), 3(c) and 3(e)] and on the outer flow domain [Figs. 3(b), 3(d) and 3(f)]. In particular, the budget terms are shown for the unfiltered field [Figs. 3(a) and 3(b)], for a filtered field with filter lengths $\lambda_{z}^{+}=76$ and $\lambda_{\varphi}^{+}=42$ [Figs. 3(c) and 3(d)], and for a filtered field with filter lengths $\lambda_{z}^{+}=9000$ and $\lambda_{\varphi}^{+}=1000$ [Figs. 3(e) and 3(f)]. Figure 3(c) reveals that all terms in the streamwise Reynolds stress budget equation lose in magnitude when small scales are omitted from velocity and pressure fields. Due to the residual stresses $\tau_{i j}$, two additional terms appear in the budget equation. The first one - presented by the yellow line in Fig. 3 - describes the transport of streamwise turbulent kinetic energy of the low-pass filtered field in physical space caused by the 

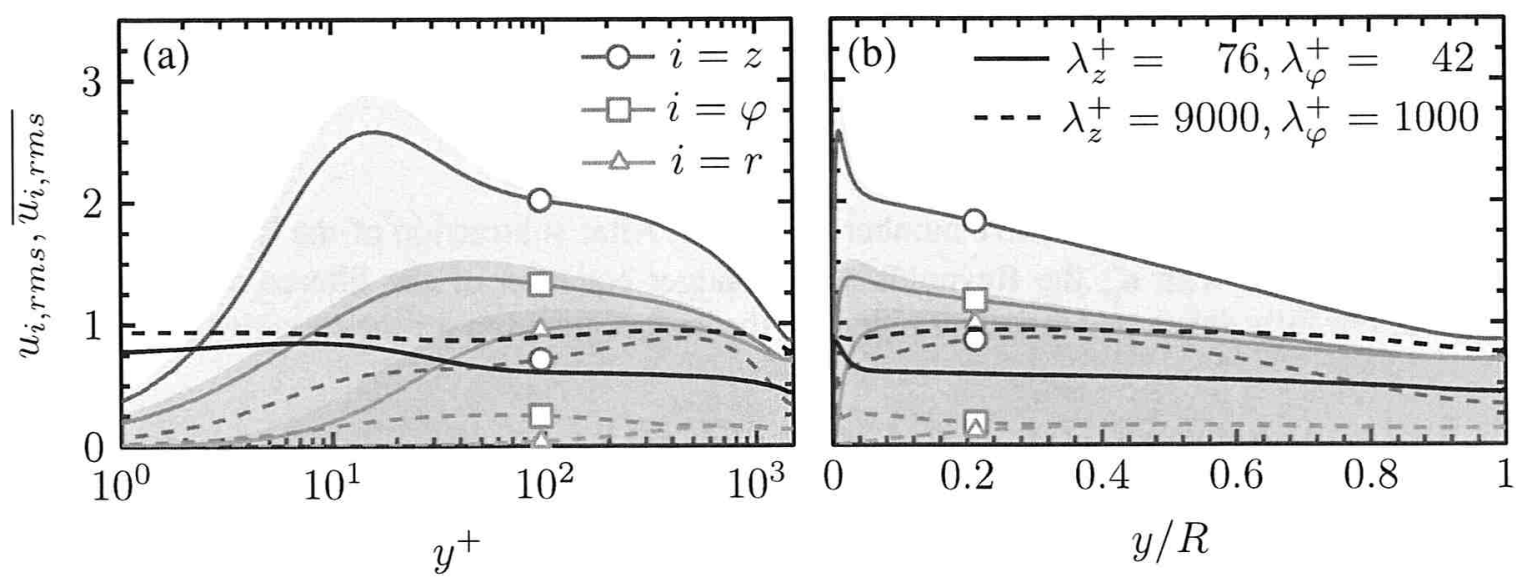

FIG. 2. Turbulent intensities for the unfiltered field (semitransparent areas), the low-pass filtered field with $\lambda_{z}^{+}=76, \lambda_{\varphi}^{+}=42$ (solid lines) and the low-pass filtered field with $\lambda_{z}^{+}=9000, \lambda_{\varphi}^{+}=1000$ (dashed lines). $-0-\overline{u_{z, r m s}} ;-\square-\overline{u_{\varphi, r m s}} ;-\triangle, \overline{u_{r, r m s}} .-$, Fraction of the streamwise component with regard to the turbulent kinetic energy $\left\langle\overline{u^{\prime}}{ }_{z} \bar{u}_{z}^{\prime}\right\rangle / \sum_{i}\left\langle\overline{u_{i}^{\prime}} \bar{u}_{i}^{\prime}\right\rangle$. (a) Focus on the near-wall region, (b) focus on the outer flow region.

interaction with smaller scales. This term is hereinafter denoted as subfilter-scale diffusion. The second additional term - sky-blue line in Fig. 3 - is commonly referred to as subgrid-scale dissipation or interscale energy flux. The term acts as a source or sink in the budget equation of the low-pass filtered field. It denotes the amount of streamwise turbulent kinetic energy that is transferred from motions smaller than the filter length to motions that are larger, and vice versa, i.e., streamwise turbulent kinetic energy that is exchanged between grid-scale and subgrid-scale motions. For the filter lengths $\lambda_{z}^{+}=76$ and $\lambda_{\varphi}^{+}=42$, the interscale energy flux term is negative throughout most of the flow domain, resulting in an average energy transfer from scales larger than the filter length into scales that are smaller. Only within a narrow region around $y^{+}=15$ the term becomes positive, meaning that energy is transferred from smaller into larger scales. This so-called backscattering or inverse energy cascade has been analyzed by Härtel et al. [6] and Piomelli et al. [7], among others. The authors connected their findings with the well-known near-wall cycle in a low Reynolds number wall-bounded flow $\left(\operatorname{Re}_{\tau}=180\right)$.

With increasing Reynolds number additional effects come into play. Outer-flow VLSM interact with the near-wall cycle, which leads to a logarithmic dependency of the near-wall statistics on the Reynolds number [4,25-29]. To efficiently analyze energy mechanisms related to VLSM, these motions are separated from near-wall small-scale motions of much higher intensity by applying filter configuration B; see Fig. 1. The terms of Eq. (6), computed with the latter filter length are shown in Figs. 3(e) and 3(f). While all budget terms of the unfiltered field [Figs. 3(a) and 3(b)] as well as the ones filtered with configuration A [Figs. 3(c) and 3(d)] are dominant very close to the wall $\left(y^{+}<100\right)$, the production term, the interscale energy flux term and the subfilter-scale diffusion term of the large-scale filtered field [Figs. 3(e) and 3(f)] are of the same order of magnitude throughout a large section in the outer flow domain. Viscous diffusion and (pseudo-)dissipation of the largest scales are dominant in the immediate vicinity of the wall $\left(y^{+}<20\right)$ and are close to zero in the rest of the flow domain. While the overall peak of production of turbulent kinetic energy is located at a wall distance of $y^{+} \approx 12$, the peak for configuration $\mathrm{B}$ is found in the logarithmic region at a wall distance of $y^{+} \approx 350$, or $y \approx 0.24 R$ in bulk units. This is a similar location as the peak of the outer-scale energy source reported by Cimarelli et al. [15]. As for the unfiltered field or configuration A filtering, the peak of production lies slightly below the peak of streamwise turbulence intensity, as presented in Fig. 2(a) $\left(y^{+} \approx 15\right)$. Ahn et al. [29] showed that large-scale motions contribute significantly to the Reynolds shear stress and, thus, to the production of turbulent kinetic energy. As Fig. 3(f) reveals, the production term appears as the only source term in the budget of the largest scales within the current framework. Unlike for a budget based on configuration A 

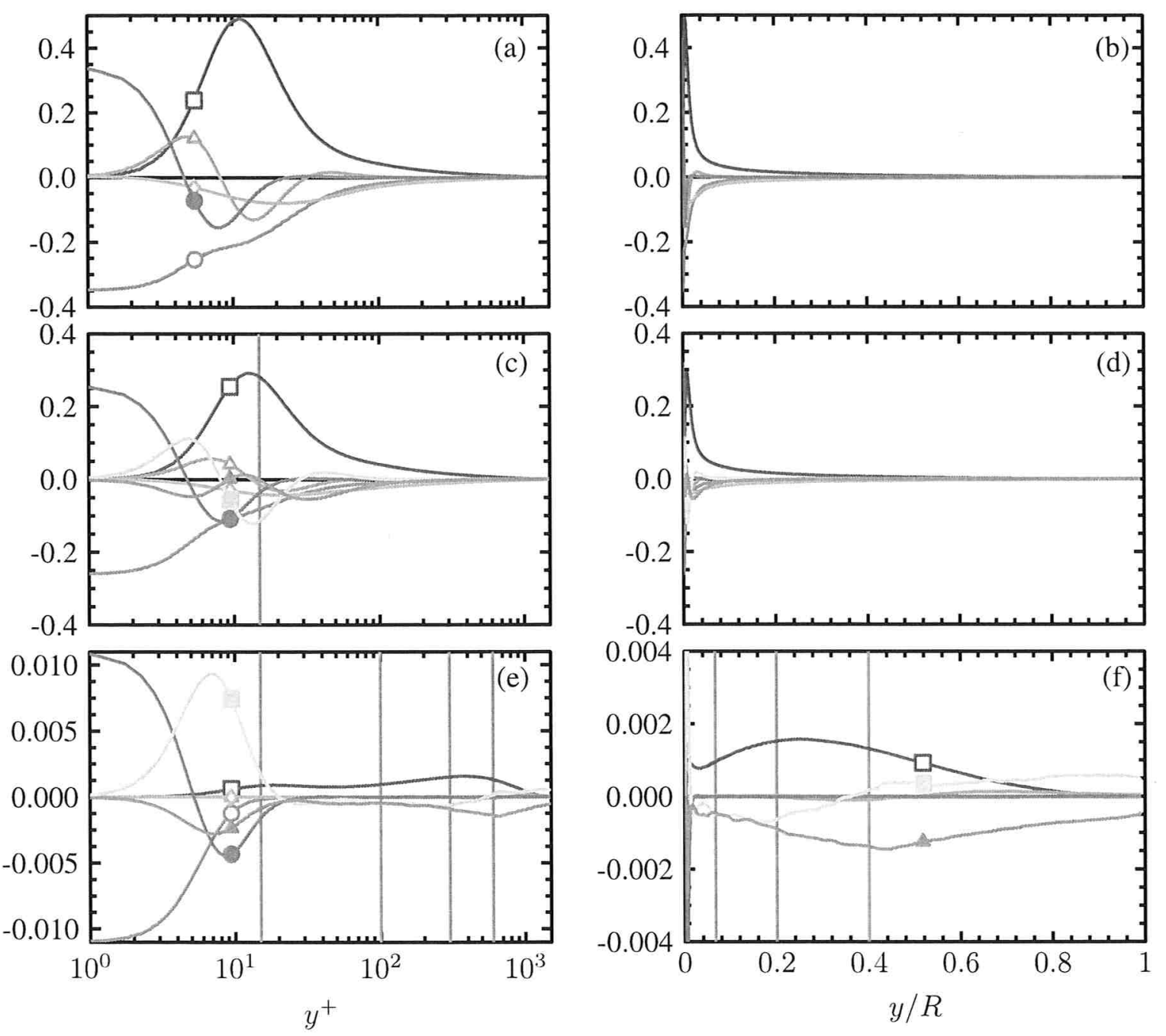

FIG. 3. Streamwise Reynolds stress budget equation terms for $\operatorname{Re}_{\tau}=1500$ of the unfiltered field (a), (b), the low-pass filtered field with $\lambda_{z}^{+}=76, \lambda_{\varphi}^{+}=42$ (c), (d) and the low-pass filtered field with $\lambda_{z}^{+}=9000, \lambda_{\varphi}^{+}=$ 1000 (e), (f). $-[-[$, production (P); $-0-$, dissipation (D); --- , viscous diffusion (VD); $\rightarrow-$, pressure-strain (PS); $-\Sigma_{-}^{-}$, turbulent diffusion (TD); - , subfilter-scale diffusion (SD); $-\mathbf{-}-$, interscale energy flux (EF). All terms normalized in viscous units. Gray lines indicate cutting planes for instantaneous flow-field realizations in Figs. 4, 7, 8, 10, 12, 15, and 16.

filtering, no mean contribution to the energy budget from smaller scales is obtained, i.e., there is no mean backscattering into VLSM. The averaged interscale energy flux stays negative over the whole wall-normal coordinate. This result expands previous findings of other studies using the GKE theory which concentrates on scale energy fluxes for scales up to $\lambda^{+}=250$ only $[10,30,31]$. On these scales the overall ( $r$-averaged) spectral-scale energy flux goes to larger scales for wall distances in the range of $y^{+}=6-37$ resembling the results of our filtering configuration $\mathrm{A}$. The spatial transport of large-scale energy away from the logarithmic region - where production exhibits its maximum toward the wall as well as toward the outer flow region is done by the subfilter-scale diffusion term as the yellow line in Fig. 3(f) indicates. In summary, smaller scales seem to play no dominant role as energy source for VLSM. However, this is only true in the mean and, as we will demonstrate in the upcoming section, we also found evidence pointing in the same direction as the findings of Cimarelli et al. [12], who described the reverse energy transfer as a crucial mechanism in the formation of very long streaks. Albeit our analysis method does not result in a mean inverse cascade for VLSM, the observation of instantaneous flow field realizations as well as conditional sampled quantities 


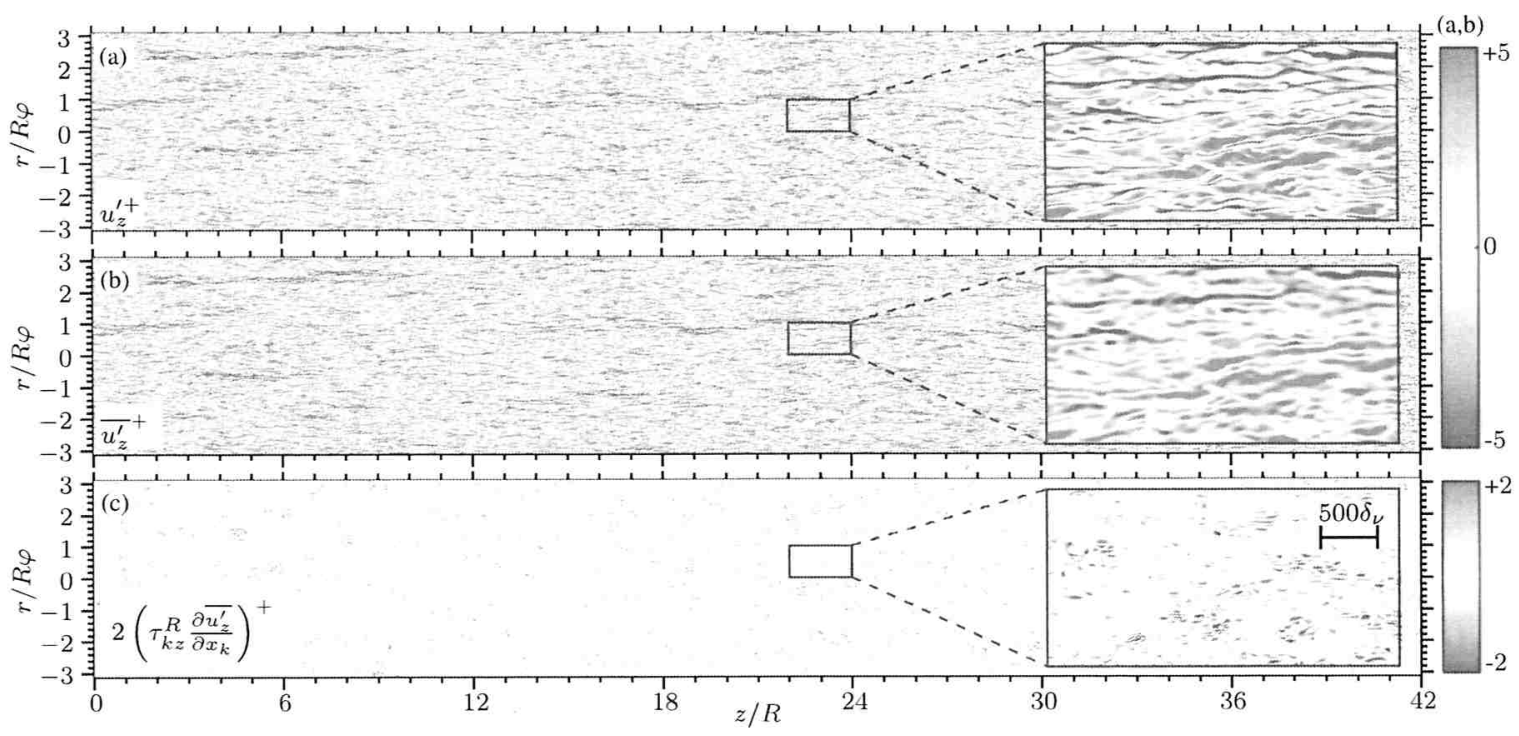

FIG. 4. Isocontours in a wall-parallel plane at $y^{+}=15$. (a) Unfiltered streamwise velocity fluctuation $u_{z}^{\prime}$. (b) Low-pass filtered streamwise velocity fluctuation $\overline{u_{z}^{\prime}}$. (c) Instantaneous interscale energy flux term, $2\left(\tau_{k z}^{R} \partial \overline{u_{z}^{\prime}} / \partial x_{k}\right)^{+}$. All terms normalized in wall units. Filter lengths: $\lambda_{z}^{+}=76, \lambda_{\varphi}^{+}=42$. The inset shows a subsection of size $l_{z}^{+} \times l_{\varphi}^{+}=3000 \times 1500$.

— carried out in Sec. IV - reveals that an inverse cascade plays indeed a role for the largest scales considered. The spatial transport of VLSM energy in the wall-normal direction is caused by interaction with subfilter scales, which will be discussed in more detail in Sec. V. Undoubtedly, VLSM receive their energy mainly from the mean field via the production term of the large-scale filtered field. Further details regarding the production term are shown in Sec. VI. In the next sections we will particularly make use of the instantaneous velocity and budget-term fields that naturally emerge from our analysis method.

\section{THE INTERSCALE ENERGY FLUX TERM}

The interscale energy flux term in the streamwise Reynolds stress budget equation of the filtered field is analyzed by means of instantaneous flow field realizations as well as by conditionally averaged one- and two-point statistics to elucidate the energy transfer mechanisms related to turbulent structures of different size and different sign. Eyink [32] derived sufficient conditions for the locality of energy transport through different scales, i.e., that energy in the turbulent cascade is transported between adjacent wave numbers. For wall-bounded flow with a low Reynolds number $\left(\operatorname{Re}_{\tau}=180\right)$ Piomelli et al. [7] showed that backscattering is related to high-speed fluid sweeping toward the solid boundary, whereas forward scattering is predominately found in regions where low-speed fluid is lifted away from the wall. Here, for filter configuration A, where filter lengths of the same order as in Piomelli et al. [7] are applied, similar results are obtained for a much larger Reynolds number of $\operatorname{Re}_{\tau}=1500$. Figure 4 shows the unfiltered and the filtered instantaneous streamwise fluctuating velocity together with the interscale energy flux for filter configuration A at a wall-distance of $y^{+}=15$. It is immediately obvious that the energy flux through the filtered scales is composed of much smaller structures than the filtered or unfiltered velocity field. The typical long and streaky structures of the velocity field cannot be observed. The correlation of backscattering events - regions of positive interscale energy flux - and high-speed regions or forward scattering and low-speed regions is not clearly visible in the instantaneous flow field realization in Fig. 4. To obtain a more conclusive image, the mean interscale energy flux is computed conditionally in the following. The condition is defined as the location in the joint probability density 


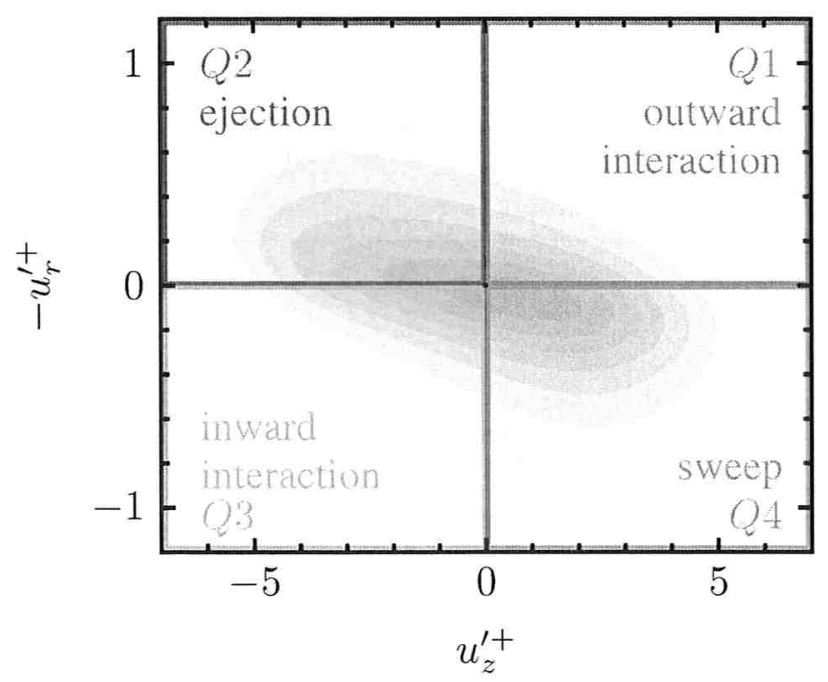

FIG. 5. Joint probability density function of the streamwise fluctuating velocity $u_{z}^{\prime+}$ and the wall-normal fluctuating velocity pointing away from the wall $-u_{r}^{\prime+}$ at a wall distance of $y^{+}=15$. Isosurfaces ranging from 0.02 (light) to 0.14 (dark) with an increment of 0.02 .

function space of the streamwise and the wall-normal velocity fluctuation, as shown in Fig. 5. The quadrant analysis has been a powerful tool in the examination of wall-bounded turbulence for several decades [33]. Here, we average the interscale energy flux separately for the different quadrants to be able to later associate the energy flux term with certain coherent structures in the fluctuating velocity field. Figure 6 depicts the conditionally averaged mean interscale energy flux obtained with filter configuration A [Fig. 6(a)] and B [Fig. 6(b)]. Figure 6(a) reveals the occurrence of backscattering in regions of $Q 4$ or sweep events $\left(u_{z}^{\prime}>0, u_{r}^{\prime}>0\right)$ in good agreement with Piomelli et al. [7]. When configuration B is applied the picture changes drastically, as will be discussed in the following. Figures 7 and 8 present the instantaneous large-scale filtered streamwise velocity field as well as the instantaneous interscale energy flux at wall distances of $y^{+}=15$ and $y^{+}=600$, respectively. As depicted in Fig. 7(c), a backscattering toward the VLSM close to the wall is observed, similar to the backscattering for wall-layer streaks. Nevertheless, now it is distinguishable by eye that backscattering is clearly related to the low-speed regions in the large-scale filtered field [Fig. 7(b)]. Forward scattering - or regions of negative interscale energy flux - on the contrary,
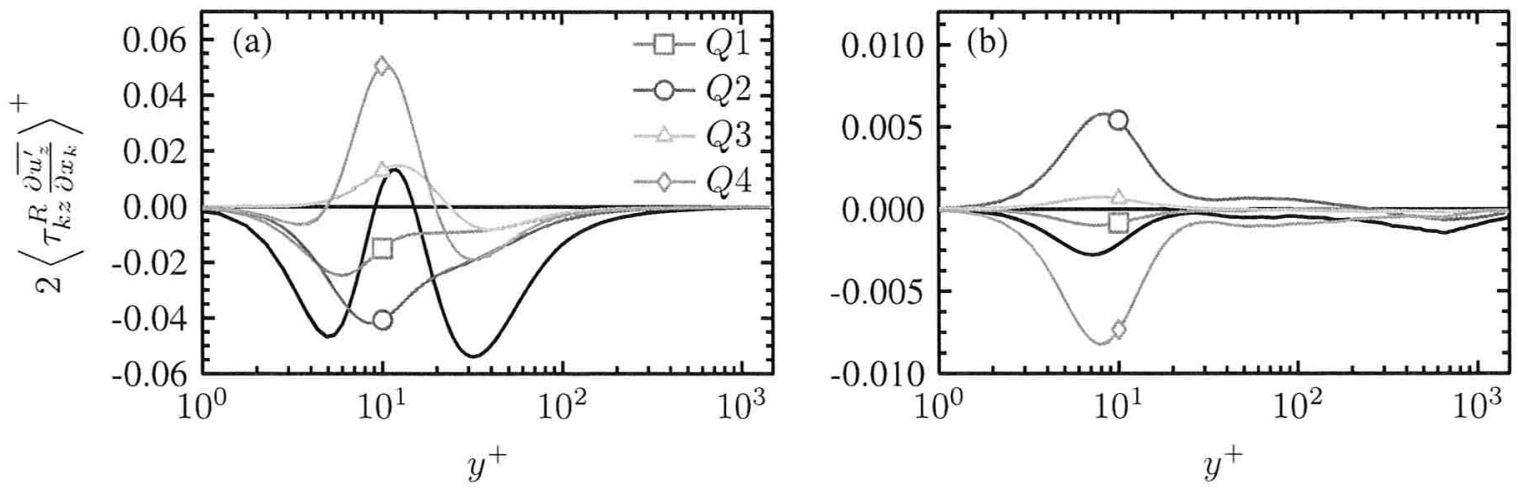

FIG. 6. Quadrantwise conditionally averaged streamwise interscale energy flux term of the low-pass filtered field $2\left\langle\tau_{k z}^{R} \partial \overline{u_{z}^{\prime}} / \partial x_{k}\right\rangle^{+}$. (a) Filter lengths $\lambda_{z}^{+}=76, \lambda_{\varphi}^{+}=42$. (b) Filter lengths $\lambda_{z}^{+}=9000, \lambda_{\varphi}^{+}=1000$. Conditions $Q 1, \overline{u_{z}^{\prime}}>0, \overline{u_{r}^{\prime}}<0 ; Q 2, \overline{u_{z}^{\prime}}<0, \overline{u_{r}^{\prime}}<0 ; Q 3, \overline{u_{z}^{\prime}}<0, \overline{u_{r}^{\prime}}>0 ; Q 4, \overline{u_{z}^{\prime}}>0, \overline{u_{r}^{\prime}}>0$. The black line shows the unconditionally averaged mean interscale energy flux as shown in Fig. 3(c). 


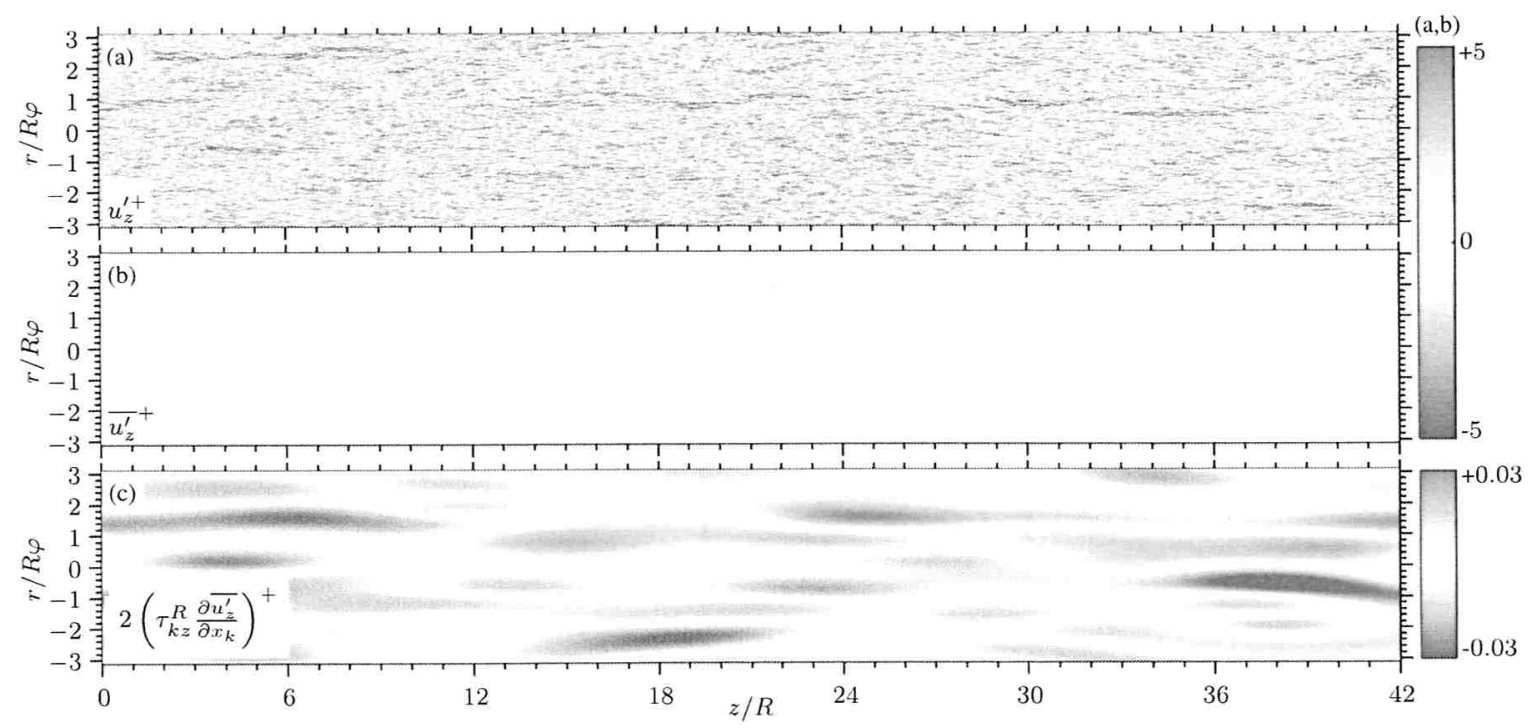

FIG. 7. Isocontours in a wall-parallel plane at $y^{+}=15$. (a) Unfiltered streamwise velocity fluctuation $u_{z}^{\prime}$. (b) Low-pass filtered streamwise velocity fluctuation $\overline{u_{z}^{\prime}}$. (c) Instantaneous interscale energy flux term, $2\left(\tau_{k z}^{R} \partial \overline{u_{z}^{\prime}} / \partial x_{k}\right)^{+}$. All terms normalized in wall units. Filter lengths: $\lambda_{z}^{+}=9000, \lambda_{\varphi}^{+}=1000$.

exhibit a strong correlation with regions of positive streamwise fluctuation in the large-scale filtered field. Further away from the wall in the outer flow region $\left(y^{+}=600\right)$, Fig. 8 shows that forward scattering is the predominant direction of energy transfer in terms of both positive and negative VLSM. These observations based on instantaneous flow field realizations are again supported by conditional averages of the interscale energy flux term, depicted in Fig. 6(b). Figure 6(b) reveals a region of strong positive (for $Q 2$ or ejection events) and negative (for $Q 4$ or sweep events) interscale energy fluxes at $y^{+}=8$, slightly below the backscattering peak obtained with filter configuration A. Somewhat weaker, but still positive the interscale energy flux related to $Q 2$ stays constant throughout most of the logarithmic layer $\left(y^{+}=30\right.$ until $\left.y^{+}=200\right)$, before it turns negative and finally collapses with the curve of sweeping VLSM and reaches a local minimum at $y^{+}=600$. It is to be noted that the forward scattering of energy away from positive (sweeping) VLSM overcompensates the backscattering into negative (ejecting) VLSM for $y^{+} \leqslant 200$, so that on average backscattering into VLSM is not observed at any distance from the wall. Structural information of the interscale energy

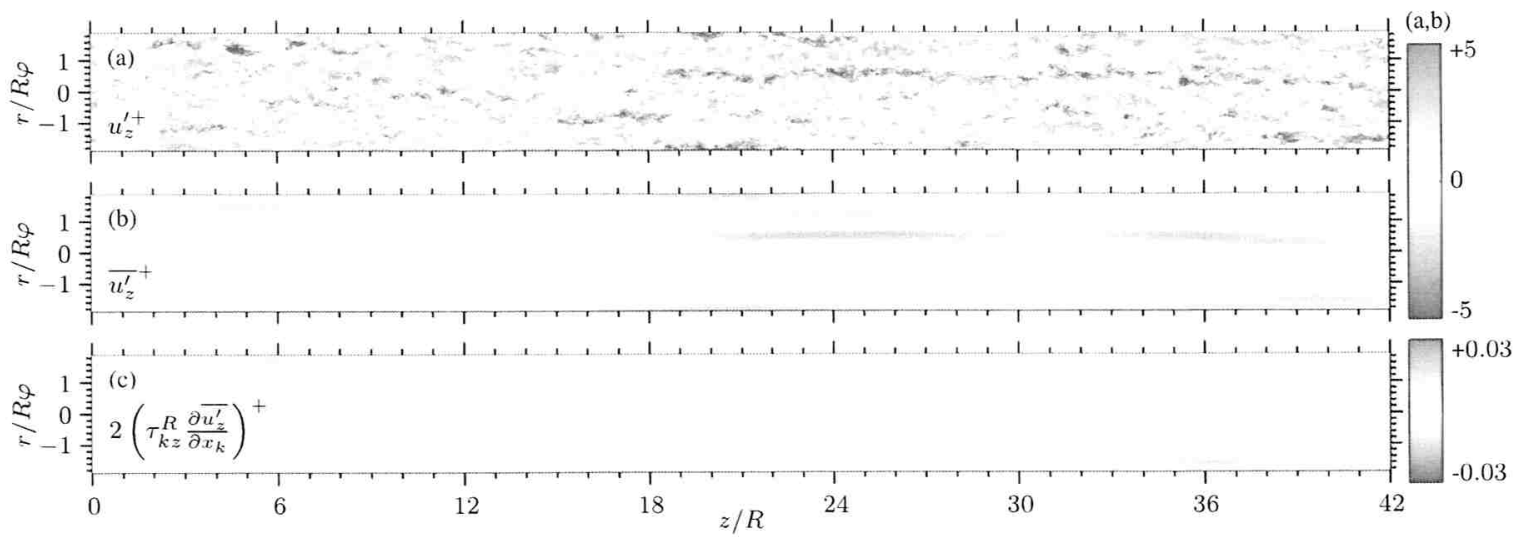

FIG. 8. Isocontours in a wall-parallel plane at $y^{+}=600$. (a) Unfiltered streamwise velocity fluctuation $u_{z}^{\prime}$. (b) Low-pass filtered streamwise velocity fluctuation $\overline{u_{z}^{\prime}}$. (c) Instantaneous interscale energy flux term, $2\left(\tau_{k z}^{R} \partial \overline{u_{z}^{\prime}} / \partial x_{k}\right)^{+}$. All terms normalized in wall units. Filter lengths: $\lambda_{z}^{+}=9000, \lambda_{\varphi}^{+}=1000$. 


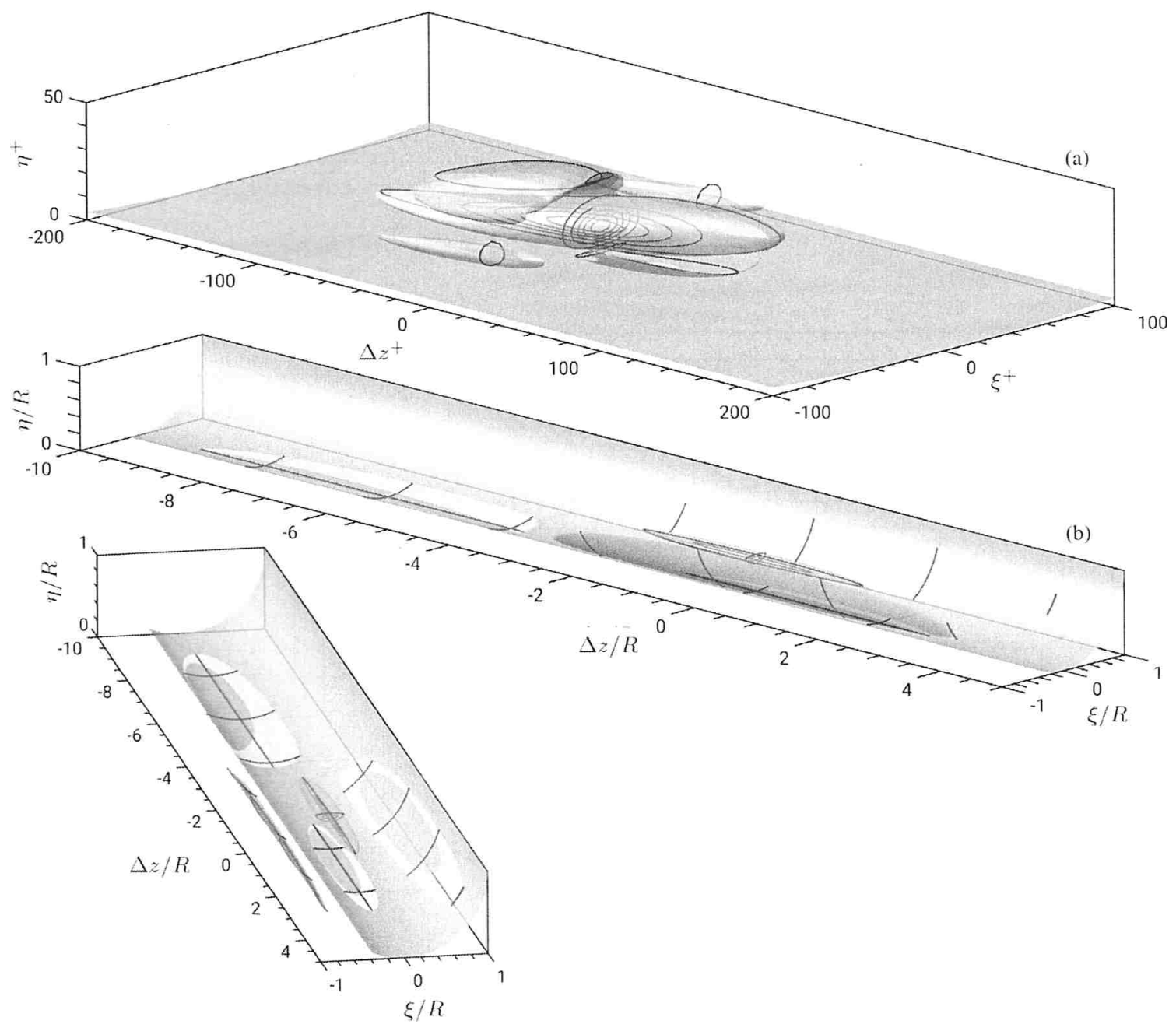

FIG. 9. Isosurfaces of the three-dimensional two-point correlation of the streamwise interscale energy flux $R_{e e}\left(\Delta z, \Delta \varphi, \Delta r, r_{0}\right)$ for configuration A (a) and B (b). (a) Reference point in the vicinity of the wall $[(R-$ $\left.r_{0}\right)^{+}=15$ ]. Orange (light-blue) isosurfaces denote a correlation of $+(-) 0.05$. The planes cutting the correlation originshow contour lines ranging from 0.05 to 0.85 (red) and from -0.05 to -0.85 (blue) with an increment of 0.2. (b) Reference point in the bulk $\left(r_{0} / R=0.6\right.$ ). Orange (light-blue) isosurfaces denote a correlation of $+(-) 0.7$. The planes cutting the correlation origin show contour lines ranging from 0.7 to 0.9 (red) and from -0.7 to -0.9 (blue) with an increment of 0.2 . Cartesian cross-sectional coordinates $\xi=\left(r_{0}+\Delta r\right) \sin (\Delta \varphi)$, $\eta=\left(r_{0}+\Delta r\right) \cos (\Delta \varphi)$.

flux can be extracted from the three-dimensional two-point correlation defined as

$$
R_{e e}\left(\Delta z, \varphi, r_{0}+\Delta r\right)=\frac{\left\langle e\left(0,0, r_{0}\right) e\left(\Delta z, \Delta \varphi, r_{0}+\Delta r\right)\right\rangle}{\left\langle e^{2}\left(0,0, r_{0}\right)\right\rangle},
$$

where $e=\tau_{1 j}^{R} \partial \overline{u_{1}^{\prime}} / \partial x_{j}$ is the instantaneous streamwise interscale energy flux. Isosurfaces of this quantity are shown in Fig. 9(a) for filter configuration $\mathrm{A}$ with a reference point near the wall $[(R-$ $\left.r_{0}\right)^{+}=15$ ] and in Fig. 9(b) for filter configuration B with a reference point in the bulk $\left(r_{0} / R=\right.$ $0.6)$. The isosurface of the interscale energy flux correlation for filter configuration A shows an inclined three-dimensional shape. The lengthy structure is accompanied by structures of opposite sign in spanwise and wall-normal directions. Since positive interscale energy flux correlates with small-scale sweeps and negative interscale energy flux with small-scale ejections, the structure of 


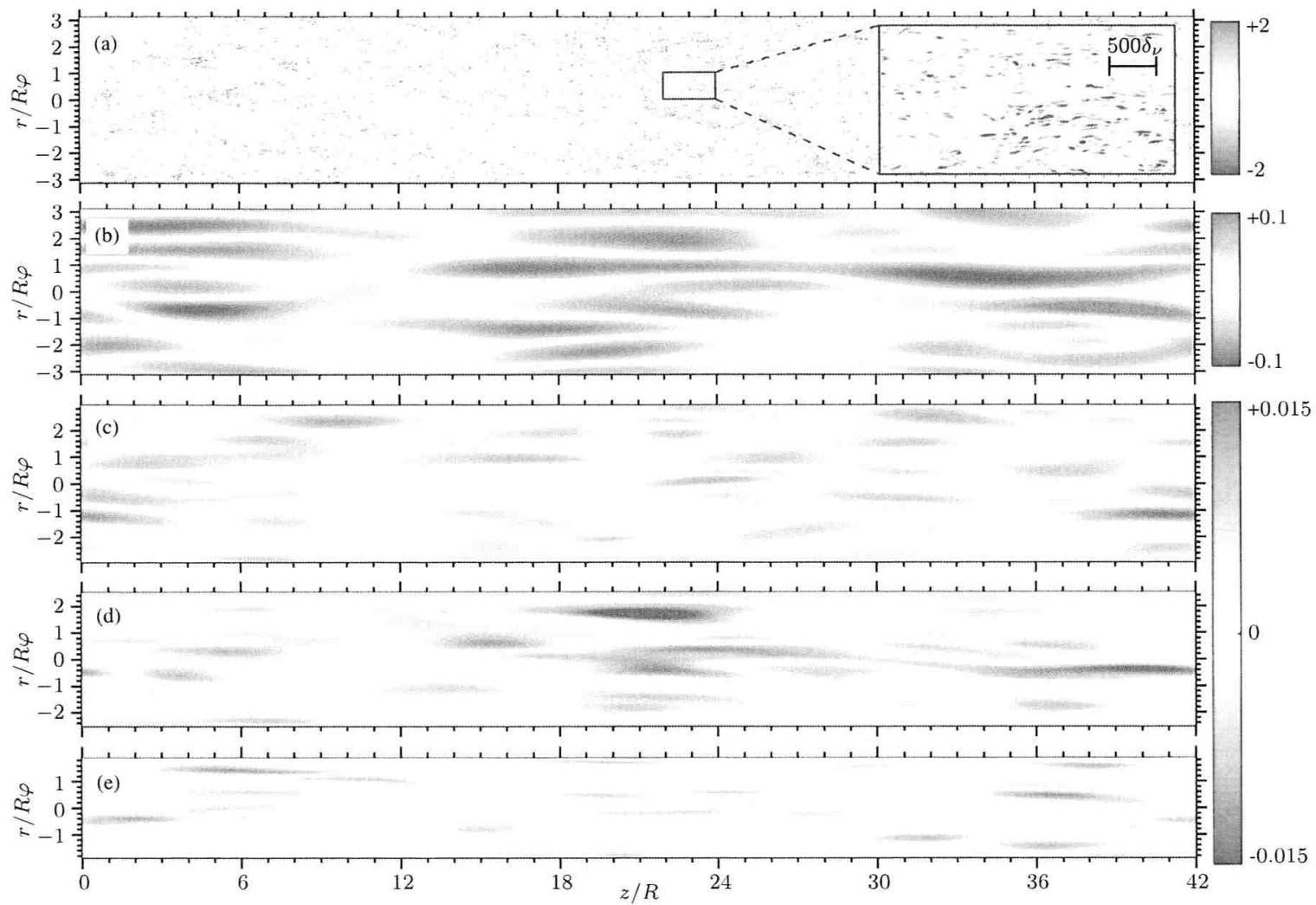

FIG. 10. Isocontours of the streamwise instantaneous subfilter-scale diffusion term $-\left[2 / r d\left(r \overline{u_{z}^{\prime}} \tau_{r z}^{R}\right) / d r\right]^{+}$ in wall-parallel planes, normalized in wall units. (a) Filter lengths: $\lambda_{z}^{+}=76, \lambda_{\varphi}^{+}=42, y^{+}=15$. (b)-(e) Filter lengths: $\lambda_{z}^{+}=9000, \lambda_{\varphi}^{+}=1000$. (a), (b) $y^{+}=15$. (c) $y^{+}=100$. (d) $y^{+}=300$. (e) $y^{+}=600$. The inset in subfigure (a) shows a subsection of size $l_{z}^{+} \times l_{\varphi}^{+}=3000 \times 1500$.

the interscale energy flux is closely related to the structure of the Reynolds shear stress, where sweeps and ejections occur in the vicinity of each other - with a spanwise spacing of approximately 120 wall units. For filter configuration B, the isosurface around the reference point seems to have "shadows" at the wall. These appear due to the fact that the interscale energy flux is intense near the wall and weak in the vicinity of the reference point. The area of negative correlation below the reference point is related to the observation of the sign reversal in the interscale energy flux term of ejecting VLSM. While very large sweeps are subject to forward scattering at all distances from the wall, very large ejections are subject to backscattering of energy near the wall and forward scattering in the bulk flow. As the small-scale motions, VLSM appear as adjacent low-speed ejections and high-speed sweeps, which reflects in the spatial arrangement of the "shadows." Here, the negative correlation structure is accompanied by positive ones in its neighborhood.

\section{THE SUBFILTER-SCALE DIFFUSION TERM}

The subfilter-scale diffusion term describes the transport of energy related to scales larger than the filter length by smaller scales in physical space. Figure 10 depicts the instantaneous term in a wall-parallel plane for the two different filter configurations and at different distances from the wall. Both strong positive and negative events in the small-scale filtered subfilter-scale diffusion field, presented in Fig. 10(a) at a wall distance of $y^{+}=15$, seem to be correlated with positive streamwise momentum regions shown in Fig. 4(b). Negative and positive events appear adjacently to each other indicating small-scale vortical motions that transport energy of the larger scales away or toward 

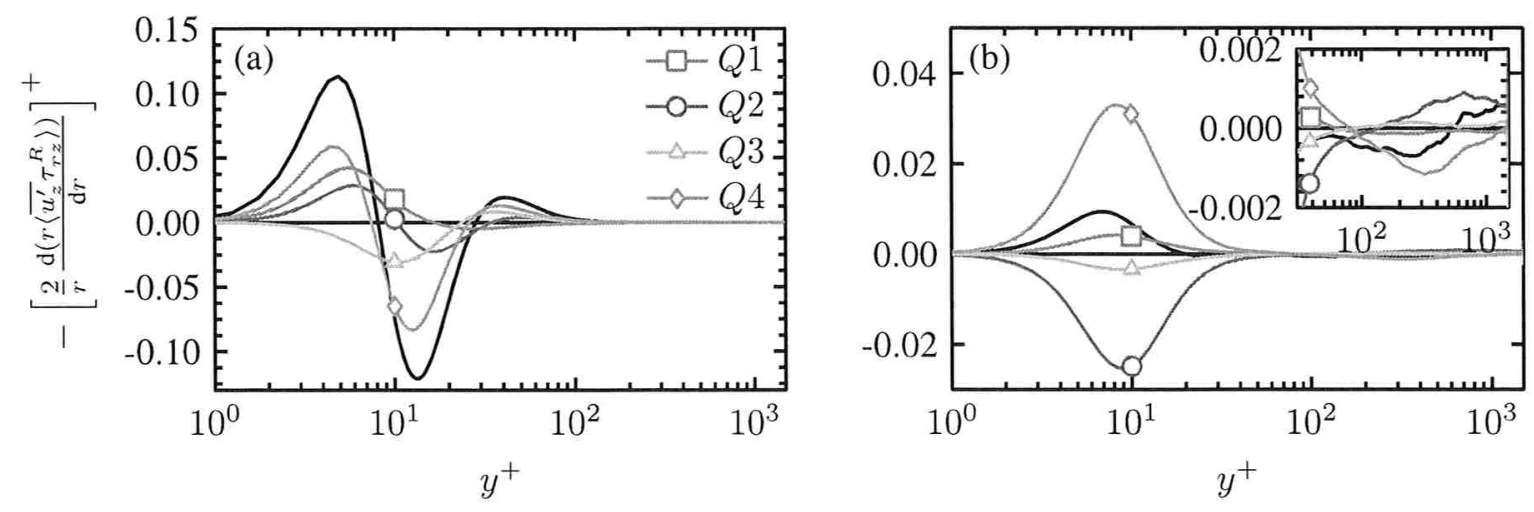

FIG. 11. Quadrantwise conditionally averaged streamwise subfilter-scale diffusion term $-\left[2 / r d\left(r\left\langle\overline{u_{z}^{\prime}} \tau_{r z}^{R}\right\rangle\right) / d r\right]^{+}$. (a) Filter lengths $\lambda_{z}^{+}=76, \lambda_{\varphi}^{+}=42$. (b) Filter lengths $\lambda_{z}^{+}=9000, \lambda_{\varphi}^{+}=1000$. Conditions as in Fig. 6 . The black line shows the unconditionally averaged mean subfilter-scale diffusion as shown in Figs. 3(c) and 3(e).

the wall distance of $y^{+}=15$. Overall, negative events occur more frequently and more intensely than positive events. This observation matches the negative mean subfilter-scale diffusion term [Fig. 3(c)] at the specific distance from the wall. Instantaneous subfilter-scale diffusion computed from the large-scale filtered field at the same wall distance [Fig. 10(b)] strongly correlates with the large-scale filtered field itself [cf. Fig. 7(b)]. Energy related to VLSM is distributed toward high-speed and away from low-speed structures through the interaction with smaller structures. When moving away from the wall, the picture becomes less clear [Figs. 10(c), 10(d) and 10(e)]. For wall distances of $y^{+}=100$ [Fig. 10(c)] and $y^{+}=600$ [Fig. 10(e)] the subfilter-scale diffusion field seems to be uncorrelated with the subfilter-scale diffusion field (as well as with the filtered velocity field) at $y^{+}=15$ [Fig. 10(b)]. However, at an intermediate wall distance of $y^{+}=300$ [Fig. 10(d)] it is clearly anticorrelated. Here in the vicinity of the mean VLSM production peak, subfilter scales transport energy from high-speed into low-speed VLSM. For comparison, filtered velocity fields at $y^{+}=100$ and $y^{+}=300$ are shown in Figs. 15 and 16, respectively. Conditionally averaged subfilter-scale diffusion terms, shown in Fig. 11, quantify the observations obtained from instantaneous fields. For filter configuration A [Fig. 11(a)], sweeps contribute most to the mean profile. This shows that the smallest scales mainly distribute energy away from the region of kinetic energy production toward the wall. Regions related to low-speed fluid ejections show a similar shape but contribute somewhat less. For filter configuration B [Fig. 11(b)], on the contrary, contributions from high- and low-speed VLSM are of the same order of magnitude but of different sign. As for filter configuration A, sweeping high-speed structures contribute to negative subfilter-scale diffusion near the peak of production $\left(y^{+} \approx 12\right.$ for small scales, $y^{+} \approx 350$ for VLSM) and to positive subfilter-scale diffusion near the wall. Ejecting low-speed structures exhibit the opposite behavior. The crossover point, where the contribution of low-speed structures becomes larger than the contribution of high-speed structures, is around $y^{+} \approx 100$. Overall, the subfilter-scale diffusion redistributes the energy spatially away from the location of maximal production toward larger and smaller wall distances. In case of large filter lengths (configuration B) and large wall distances the ejection events are fuelled by this term, while the sweep events lose energy. One possible explanation for the behavior of the subfilter-scale diffusion term for filter configuration B could be the large- but subfilter-scale vortical motions or vortex packages as described by Adrian [34] smaller than VLSM and therefore filtered out but larger than the near-wall small scales. These vortex packages are coherently aligned with the VLSM in vertical direction. Where low-speed VLSM are ejected, they transport energy from the wall into the bulk and where high-speed VLSM sweep toward the wall, they transport energy from the bulk into the near-wall region. 


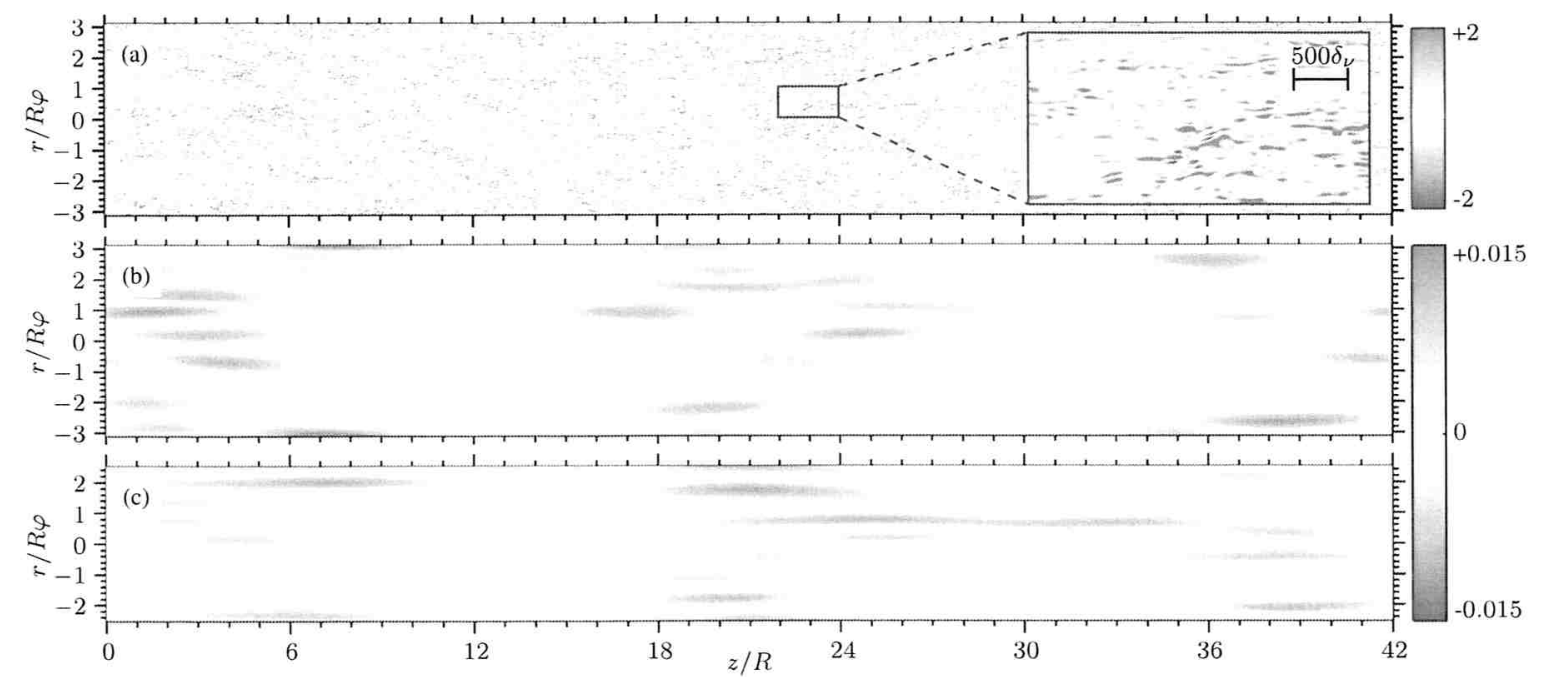

FIG. 12. Isocontours of the streamwise instantaneous production term of the filtered field $-2\left[\overline{u_{z}^{\prime}} \cdot \overline{u_{r}^{\prime}} \frac{d\left\langle u_{z}\right\rangle}{d r}\right]^{+}$ in wall-parallel planes, normalized in wall units. (a) Filter lengths: $\lambda_{z}^{+}=76, \lambda_{\varphi}^{+}=42$. (b), (c) Filter lengths: $\lambda_{z}^{+}=9000, \lambda_{\varphi}^{+}=1000$. (a), (b) $y^{+}=15$. (c) $y^{+}=300$. The inset in subfigure (a) shows a subsection of size $l_{z}^{+} \times l_{\varphi}^{+}=3000 \times 1500$.

\section{THE PRODUCTION TERM OF THE FILTERED FIELD}

Instantaneous realizations of the production term of the low-pass filtered field are shown in Fig. 12 for filter configuration A [Fig. 12(a)] and B [Figs. 12(b) and 12(c)] in wall-parallel planes at wall distances of $y^{+}=15$ [Figs. 12(a) and 12(b)] and $y^{+}=300$ [Fig. 12(c)]. The former wall distance is related to the peak of production of kinetic energy of the full field [cf. Fig. 3(a)] or the small-scale filtered field [cf. Fig. 3(c)]. The latter wall distance is located in the vicinity of the production peak of the large-scale filtered field [cf. Fig. 3(e)]. The production term is on average always positive, which means that it acts as a source of energy in the transport equation of the fluctuating field and as a sink in the transport equation of the mean field. Instantaneously, regions of negative production are found in the vicinity of the wall both for filter configuration $\mathrm{A}$ [Fig. 12(a)] and filter configuration B [Fig. 12(b)], which indicates that the fluctuating field also energizes the mean field locally. However, positive contributions to the mean production term are more dominant. The comparison of Fig. 12(a) with the small-scale filtered streamwise velocity field [Fig. 4(b)] shows that the strongest instantaneous production events correlate with regions of high-speed motions. The production term computed with filter configuration B features negative regions covering a large area of the wall-normal plane at $y^{+}=15$ [Fig. 12(b)]. Consequently, VLSM do not only receive energy from the mean field via the production term in the vicinity of the wall but they also release it. At $y^{+}=300$, near the production peak of the large-scale filtered field, the instantaneous production field presented in Fig. 12(c) exhibits almost only positive regions. The elongated regions of intense production events can be related to either low-speed or high-speed VLSM, as they are visible in the low-pass filtered streamwise velocity field at the same distance from the wall (see Fig. 16 in the Appendices). Again, the conditionally averaged production term of the streamwise energy budget of the low-pass filtered field, shown in Fig. 13, validates the instantaneous field observations. High-speed sweeps contribute somewhat more to the production term of the small-scale filtered field [Fig. 13(a)] than ejections. $Q 1$ and $Q 3$ events - inward and outward interactions, respectively - exhibit negative but little contributions to the overall production profile. The production profiles obtained from filter configuration B collapse for sweeps and ejections [Fig. 13(b)]. Therefore, $Q 1$ and $Q 3$ events do not play a significant role in terms of the turbulent production profile of VLSM. In the vicinity of the wall the $Q 4$ - and $Q 2$-conditioned 

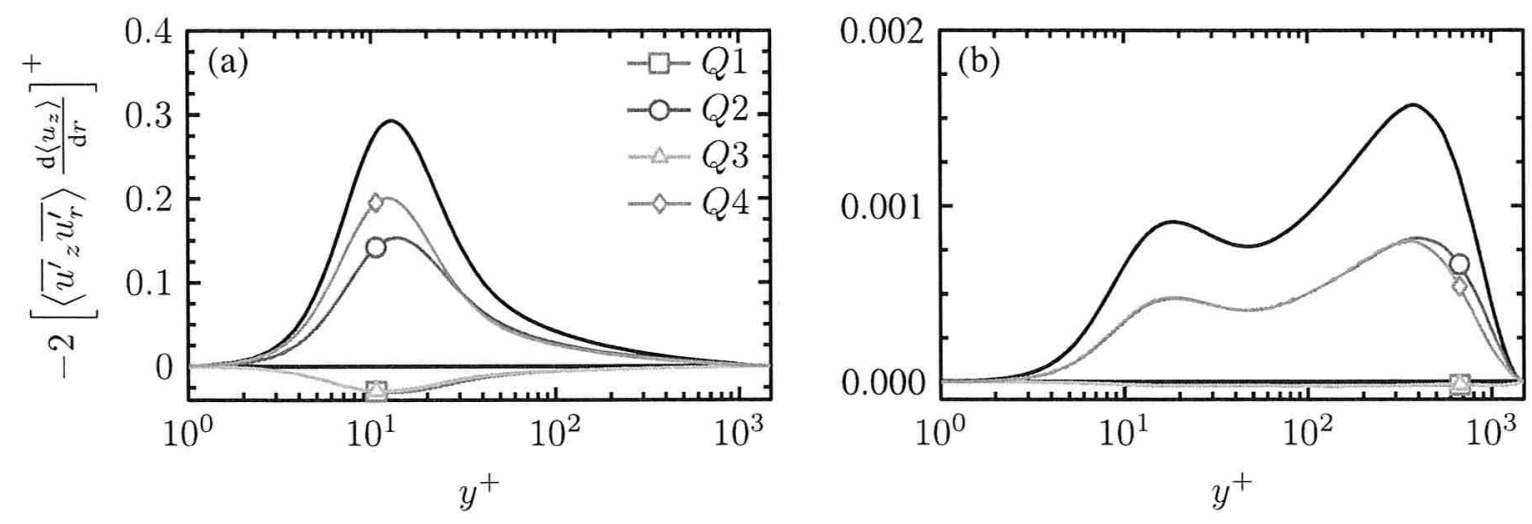

FIG. 13. Quadrantwise conditionally averaged streamwise production term of the low-pass filtered field $-2\left[\left\langle\overline{u_{z}^{\prime}} \overline{u_{r}^{\prime}}\right\rangle \frac{d\left\langle u_{z}\right\rangle}{d r}\right]^{+}$. (a) Filter lengths $\lambda_{z}^{+}=76, \lambda_{\varphi}^{+}=42$. (b) Filter lengths $\lambda_{z}^{+}=9000, \lambda_{\varphi}^{+}=1000$. Conditions as in Fig. 6 . The black line shows the unconditionally averaged mean production of the filtered field as shown in Figs. 3(c) and 3(e).

profiles collapse, whereas in the outer flow region above $y^{+} \approx 300$, the profile related to ejections is of a marginally larger level than the sweep profile.

\section{CONCLUSION}

The analysis of the streamwise budget equation of the low-pass filtered velocity field revealed the energy transfer mechanisms related to different scales in turbulent pipe flow. Only three dominant terms persist throughout most of the flow domain when the budget equation is evaluated for a flow field where all streamwise scales smaller than VLSM have been filtered out. These are the production term, the interscale energy flux term and the subfilter-scale diffusion term. Only in the very vicinity of the wall do viscous diffusion and dissipation play a role for VLSM. The production term of the low-pass filtered field is found to be the key energy source of VLSM. Its peak is located at the end of the logarithmic region around $y^{+} \approx 350$. The subfilter-scale diffusion term transports the energy related to VLSM further toward the bulk and closer to the wall. The average interscale energy flux term transfers energy into smaller scales. The first two observations show similarity to the near-wall small-scale cycle, where the peak of production is located at $y^{+} \approx 15$ and the subfilter-scale diffusion term redistributes energy together with the other transport terms toward the bulk and the wall. The interscale energy flux, on the contrary, is not only dissipative for the small scales, but exhibits backscattering around $y^{+}=15$, where turbulent sweeps and ejections are found. Conditional averages confirm earlier observations by Piomelli et al. [7] that backscattering toward the small-scale near-wall motions is related to sweeps, whereas forward scattering correlates with ejections. Surprisingly, when applying filter configuration B, the opposite result is obtained for VLSM. Here, backscattering is correlated with ejections and forward scattering with sweeps. In both cases sweeps are the more intense events, so that on average a positive interscale energy flux and, thus, backscattering is obtained for filter configuration $\mathrm{A}$ in the flow region populated by small-scale wall-layer structures, whereas for filter configuration B the mean interscale energy flux stays negative throughout the whole flow domain. In general, backscattering takes place in regions of large turbulent kinetic energy production. The correlation between the direction of the interscale energy flux and the direction of fluid motion in space suggests that these coherent structures are subject to backscattering, which enter these regions of maximum production. With respect to the small scales these structures are high-speed sweeps entering the near-wall region, whereas for VLSM low-speed fluid is ejected toward the outer production peak in the logarithmic region. The correlation between very-large-scale ejection events and reverse energy flux (filter configuration B), determined using conditional averages, might be viewed as an extension of the spatial reverse cascades of Cimarelli et al. $[12,15]$ involved in the creation of very-large-scale velocity fluctuations in the outer flow 
regions. Our analysis produces instantaneous interscale energy flux fields as well as conditionally sampled quantities and two-point correlations, which provide a potential structural picture of the interscale energy transfer. The subfilter-scale diffusion term describes the energy transport of scales larger than the filter lengths by the smaller scales. For both filter configurations energy is transported from the wall distance of maximum turbulent production of the corresponding scale $\left(y^{+} \approx 15\right.$ or $y^{+} \approx 350$ ) toward the wall and the bulk. While both sweeps and ejections contribute in the same manner to the mean subfilter-scale diffusion profile of the small-scale filtered field, they play contradicting roles in the profile of the large-scale filtered field. Within high-speed VLSM or very large sweeps energy is transported away from the vicinity of the large-scale production peak $\left(y^{+} \approx 350\right)$ toward the near-wall region $\left(y^{+} \approx 8\right)$ by the subfilter-scale diffusion term. Low-speed VLSM or very large ejections, on the contrary, are related to a subfilter-scale diffusion from the near-wall region $\left(y^{+} \approx 8\right)$ to the outer flow region $\left(y^{+} \approx 650\right)$. The contribution of the subfilter-scale diffusion term to the budget of filter configuration $\mathrm{B}$ might be related to large-scale vortices or vortex packages smaller than the filter lengths that are coherently aligned with the VLSM, which are larger than the filter lengths. This behavior of large- and very-large-scale motions is strikingly similar to the well-known near-wall cycle of small-scale wall-layer streaks and quasi-streamwise vortices. For the case of turbulent plane channel flow Hwang and Cossu [35-37] argued that large-scale motions even self-sustain, when the small scales are artificially removed. Feldmann and Avila [38] performed a similar analysis for turbulent pipe flow. They interpreted that the artificial removal of small scales is analogous to a reduction of the Reynolds number, where the sustaining large-scale motions represent the near-wall cycle. Kawata and Alfredsson [22], on the contrary, found that inverse interscale energy transport of Reynolds shear stress is responsible for the turbulent kinetic energy production at large scales in turbulent plane Couette flow. The present study of the dominant terms of the streamwise energy budget of the low-pass filtered field supports the interaction of small scales with VLSM in turbulent pipe flow, especially by the action of the subfilter-scale diffusion term. Further, our work corroborates recent findings [15] that the overall picture of energy fluxes in turbulent flow involves the coexistence of direct and inverse cascades at different scales. Our analysis method allows us to identify the cascades and fluxes belonging to particular events, such as sweeps and ejections, and thus expands other recent studies on turbulent plane-channel flow $[16,17]$.

\section{ACKNOWLEDGMENTS}

The authors gratefully acknowledge the Gauss Centre for Supercomputing e.V. (www. gauss-centre.eu) for funding this project by providing computing time on the GCS Supercomputer SuperMUC at Leibniz Supercomputing Centre (www.lrz.de). Moreover, the authors thank Annika Köhne for proof-reading.

\section{APPENDIX A: DERIVATION OF THE REYNOLDS STRESS TRANSPORT EQUATION FOR A FILTERED VELOCITY FIELD}

Applying the filtering operation Eq. (4) to the dimensionless momentum Eq. (1) results in

$$
\frac{\partial \bar{u}_{j}}{\partial t}+\frac{\partial \overline{u_{i} u_{j}}}{\partial x_{i}}=\frac{1}{\operatorname{Re}_{\tau}} \frac{\partial^{2} \bar{u}_{j}}{\partial x_{i} \partial x_{i}}-\frac{\partial \bar{p}}{\partial x_{j}} .
$$

In addition, the averaging operation Eq. (3) is applied to the momentum Eq. (1) to obtain the Reynolds equation

$$
\begin{gathered}
\frac{\partial\left\langle u_{j}\right\rangle}{\partial t}+\frac{\partial\left\langle u_{i} u_{j}\right\rangle}{\partial x_{i}}=\frac{1}{\operatorname{Re}_{\tau}} \frac{\partial^{2}\left\langle u_{j}\right\rangle}{\partial x_{i} \partial x_{i}}-\frac{\partial\langle p\rangle}{\partial x_{j}} \\
\Leftrightarrow \frac{\partial\left\langle u_{j}\right\rangle}{\partial t}+\frac{\partial\left\langle u_{i}\right\rangle\left\langle u_{j}\right\rangle}{\partial x_{i}}=-\frac{\partial\left\langle u_{i}^{\prime} u_{j}^{\prime}\right\rangle}{\partial x_{i}}+\frac{1}{\operatorname{Re}_{\tau}} \frac{\partial^{2}\left\langle u_{j}\right\rangle}{\partial x_{i} \partial x_{i}}-\frac{\partial\langle p\rangle}{\partial x_{j}} . \\
064607-16
\end{gathered}
$$


Subtracting the Reynolds Eq. (A2) from the filtered momentum Eq. (A1) provides an equation for the filtered fluctuating velocity

$$
\begin{aligned}
& \frac{\partial\left(\bar{u}_{j}-\left\langle u_{j}\right\rangle\right)}{\partial t}+\frac{\partial\left(\overline{u_{i} u_{j}}-\left\langle u_{i}\right\rangle\left\langle u_{j}\right\rangle\right)}{\partial x_{i}}=\frac{\partial\left\langle u_{i}^{\prime} u_{j}^{\prime}\right\rangle}{\partial x_{i}}+\frac{1}{\operatorname{Re}_{\tau}} \frac{\partial^{2}\left(\bar{u}_{j}-\left\langle u_{j}\right\rangle\right)}{\partial x_{i} \partial x_{i}}-\frac{\partial(\bar{p}-\langle p\rangle)}{\partial x_{j}} \\
\Leftrightarrow & \frac{\partial \overline{\left(u_{j}-\left\langle u_{j}\right\rangle\right)}}{\partial t}+\frac{\partial \overline{\left(u_{i} u_{j}-\left\langle u_{i}\right\rangle\left\langle u_{j}\right\rangle\right)}}{\partial x_{i}}=\frac{\partial\left\langle u_{i}^{\prime} u_{j}^{\prime}\right\rangle}{\partial x_{i}}+\frac{1}{\operatorname{Re}_{\tau}} \frac{\partial^{2} \overline{\left(u_{j}-\left\langle u_{j}\right\rangle\right)}}{\partial x_{i} \partial x_{i}}-\frac{\partial \overline{(p-\langle p\rangle)}}{\partial x_{j}} \\
\Leftrightarrow & \frac{\partial \overline{u_{j}^{\prime}}}{\partial t}+\frac{\partial\left(\overline{\left\langle u_{i}\right\rangle u_{j}^{\prime}}+\overline{u_{i}^{\prime}\left\langle u_{j}\right\rangle}+\overline{u_{i}^{\prime} u_{j}^{\prime}}\right)}{\partial x_{i}}=\frac{\partial\left\langle u_{i}^{\prime} u_{j}^{\prime}\right\rangle}{\partial x_{i}}+\frac{1}{\operatorname{Re}_{\tau}} \frac{\partial^{2} \overline{u_{j}^{\prime}}}{\partial x_{i} \partial x_{i}}-\frac{\partial \overline{p^{\prime}}}{\partial x_{j}} .
\end{aligned}
$$

In analogy to Pope [24], p. $581 \mathrm{ff}$, we define a residual stress tensor

$$
\tau_{i j}^{R}=\overline{u_{i} u_{j}}-\overline{u_{i}} \cdot \overline{u_{j}}=\overline{u_{i}^{\prime} u_{j}^{\prime}}-\overline{u_{i}^{\prime}} \cdot \overline{u_{j}^{\prime}} .
$$

We apply the latter expression and substitute index $i$ by $k$ in Eq. (A3) yielding

$$
\frac{\partial \overline{u_{j}^{\prime}}}{\partial t}+\overline{u_{k}^{\prime}} \frac{\partial \overline{u_{j}^{\prime}}}{\partial x_{k}}=\frac{1}{\operatorname{Re}_{\tau}} \frac{\partial^{2} \overline{u_{j}^{\prime}}}{\partial x_{k} \partial x_{k}}+\frac{\partial}{\partial x_{k}}\left(\left\langle u_{k}^{\prime} u_{j}^{\prime}\right\rangle-\left\langle u_{k}\right\rangle \overline{u_{j}^{\prime}}-\overline{u_{k}^{\prime}}\left\langle u_{j}\right\rangle-\tau_{k j}^{R}\right)-\frac{\partial \overline{p^{\prime}}}{\partial x_{j}} .
$$

Now, we multiply Eq. (A5) for component $i$ and $j$ with $\overline{u_{j}^{\prime}}$ and $\overline{u_{i}^{\prime}}$, respectively, to obtain

$$
\begin{aligned}
& \overline{u_{i}^{\prime}} \frac{\partial \overline{u_{j}^{\prime}}}{\partial t}+\overline{u_{i}^{\prime}} \cdot \overline{u_{k}^{\prime}} \frac{\partial \overline{u_{j}^{\prime}}}{\partial x_{k}}=\frac{1}{\operatorname{Re}_{\tau}} \overline{u_{i}^{\prime}} \frac{\partial^{2} \overline{u_{j}^{\prime}}}{\partial x_{k} \partial x_{k}}+\overline{u_{i}^{\prime}} \frac{\partial}{\partial x_{k}}\left(\left\langle u_{k}^{\prime} u_{j}^{\prime}\right\rangle-\left\langle u_{k}\right\rangle \overline{u_{j}^{\prime}}-\overline{u_{k}^{\prime}}\left\langle u_{j}\right\rangle-\tau_{k j}^{R}\right)-\overline{u_{i}^{\prime}} \frac{\partial \overline{p^{\prime}}}{\partial x_{j}}, \\
& \overline{u_{j}^{\prime}} \frac{\partial \overline{u_{i}^{\prime}}}{\partial t}+\overline{u_{j}^{\prime}} \cdot \overline{u_{k}^{\prime}} \frac{\partial \overline{u_{i}^{\prime}}}{\partial x_{k}}=\frac{1}{\operatorname{Re}_{\tau}} \overline{u_{j}^{\prime}} \frac{\partial^{2} \overline{u_{i}^{\prime}}}{\partial x_{k} \partial x_{k}}+\overline{u_{j}^{\prime}} \frac{\partial}{\partial x_{k}}\left(\left\langle u_{k}^{\prime} u_{i}^{\prime}\right\rangle-\left\langle u_{k}\right\rangle \overline{u_{i}^{\prime}}-\overline{u_{k}^{\prime}}\left\langle u_{i}\right\rangle-\tau_{k i}^{R}\right)-\overline{u_{j}^{\prime}} \frac{\partial \overline{p^{\prime}}}{\partial x_{j}} .
\end{aligned}
$$

The sum of Eqs. (A6) and (A7) leads to the expression

$$
\begin{aligned}
& \frac{\partial\left(\overline{u_{i}^{\prime}} \cdot \overline{u_{j}^{\prime}}\right)}{\partial t}+\underbrace{\overline{u_{k}^{\prime}} \frac{\partial\left(\overline{u_{i}^{\prime}} \cdot \overline{u_{j}^{\prime}}\right)}{\partial x_{k}}=}_{(i)}=\underbrace{\frac{1}{\operatorname{Re}_{\tau}}\left(\overline{u_{i}^{\prime}} \frac{\partial^{2} \overline{u_{j}^{\prime}}}{\partial x_{k} \partial x_{k}}+\overline{u_{j}^{\prime}} \frac{\partial^{2} \overline{u_{i}^{\prime}}}{\partial x_{k} \partial x_{k}}\right)+\overline{u_{i}^{\prime}} \frac{\partial\left\langle u_{k}^{\prime} u_{j}^{\prime}\right\rangle}{\partial x_{k}}+\overline{u_{j}^{\prime}} \frac{\partial\left\langle u_{k}^{\prime} u_{i}^{\prime}\right\rangle}{\partial x_{k}}}_{(i i)} \\
&-\underbrace{\overline{u_{i}^{\prime}} \frac{\partial\left\langle u_{k}\right\rangle \overline{u_{j}^{\prime}}}{\partial x_{k}}-\overline{u_{j}^{\prime}} \frac{\partial\left\langle u u_{k}\right\rangle \overline{u_{i}^{\prime}}}{\partial x_{k}}-\overline{u_{i}^{\prime}} \frac{\partial \overline{u_{k}^{\prime}}\left\langle u_{j}\right\rangle}{\partial x_{k}}-\overline{u_{j}^{\prime}} \frac{\partial \overline{u_{k}^{\prime}}\left\langle u u_{i}\right\rangle}{\partial x_{k}}}_{(i i i)} \\
&-\overline{u_{i}^{\prime}} \frac{\partial \tau_{k j}^{R}}{\partial x_{k}}-\overline{u_{j}^{\prime}} \frac{\partial \tau_{k i}^{R}}{\partial x_{k}}-\overline{u_{i}^{\prime}} \frac{\partial \overline{p^{\prime}}}{\partial x_{j}}-\overline{u_{j}^{\prime}} \frac{\partial \overline{p^{\prime}}}{\partial x_{i}} .
\end{aligned}
$$

Term (i) is rewritten to

$$
\overline{u_{k}^{\prime}} \frac{\partial\left(\overline{u_{i}^{\prime}} \cdot \overline{u_{j}^{\prime}}\right)}{\partial x_{k}}=\frac{\partial\left(\overline{u_{i}^{\prime}} \cdot \overline{u_{j}^{\prime}} \cdot \overline{u_{k}^{\prime}}\right)}{\partial x_{k}}-\left(\overline{u_{j}^{\prime}} \cdot \overline{u_{j}^{\prime}}\right) \frac{\partial \overline{u_{k}^{\prime}}}{\partial x_{k}}
$$


The first term on the right-hand side of Eq. (A8) (ii) can be simplified as follows:

$$
\begin{aligned}
\frac{1}{\operatorname{Re}_{\tau}}\left(\overline{u_{i}^{\prime}} \frac{\partial^{2} \overline{u_{j}^{\prime}}}{\partial x_{k} \partial x_{k}}+\overline{u_{j}^{\prime}} \frac{\partial^{2} \overline{u_{i}^{\prime}}}{\partial x_{k} \partial x_{k}}\right) & =\frac{1}{\operatorname{Re}_{\tau}}\left(\frac{\partial}{\partial x_{k}}\left(\overline{u_{i}^{\prime}} \frac{\partial \overline{u_{j}^{\prime}}}{\partial x_{k}}\right)-\frac{\partial \overline{u_{i}^{\prime}}}{\partial x_{k}} \frac{\partial \overline{u_{j}^{\prime}}}{\partial x_{k}}+\frac{\partial}{\partial x_{k}}\left(\overline{u_{j}^{\prime}} \frac{\partial \overline{u_{i}^{\prime}}}{\partial x_{k}}\right)-\frac{\partial \overline{u_{j}^{\prime}}}{\partial x_{k}} \frac{\partial \overline{u_{i}^{\prime}}}{\partial x_{k}}(\rangle\right. \\
& =\frac{1}{\operatorname{Re}_{\tau}}\left(-2 \frac{\partial \overline{u_{j}^{\prime}}}{\partial x_{k}} \frac{\partial \overline{u_{i}^{\prime}}}{\partial x_{k}}+\frac{\partial^{2} \overline{u_{i}^{\prime}} \cdot \overline{u_{j}^{\prime}}}{\partial x_{k} \partial x_{k}}\right) \\
& =-\frac{2}{\operatorname{Re}_{\tau}}\left(\frac{\partial \overline{u_{j}^{\prime}}}{\partial x_{k}} \frac{\partial \overline{u_{i}^{\prime}}}{\partial x_{k}}\right)+\frac{1}{\operatorname{Re}_{\tau}} \frac{\left.\partial^{2} \overline{\left(\overline{u_{i}^{\prime}}\right.} \cdot \overline{u_{j}^{\prime}}\right)}{\partial x_{k} \partial x_{k}} .
\end{aligned}
$$

With the aid of the reformulations

$$
\overline{u_{\ell}^{\prime}} \frac{\partial\left(\overline{u_{k}^{\prime}}\left\langle u_{m}\right\rangle\right)}{\partial x_{k}}=\overline{u_{\ell}^{\prime}}\left(\overline{u_{k}^{\prime}} \frac{\partial\left\langle u_{m}\right\rangle}{\partial x_{k}}+\left\langle u_{m}\right\rangle \frac{\partial \overline{u_{k}}}{\partial x_{k}}\right)=\overline{u_{\ell}^{\prime}} \cdot \overline{u_{k}^{\prime}} \frac{\partial\left\langle u_{m}\right\rangle}{\partial x_{k}}
$$

and

$$
\overline{u_{\ell}^{\prime}} \frac{\partial\left(\left\langle u_{k}\right\rangle \overline{u_{m}^{\prime}}\right)}{\partial x_{k}}=\overline{u_{\ell}^{\prime}}\left(\left\langle u_{k}\right\rangle \frac{\partial \overline{u_{m}^{\prime}}}{\partial x_{k}}+\overline{u_{m}^{\prime}} \frac{\partial\left\langle u_{k}\right\rangle}{\partial x_{k}}\right)=\overline{u_{\ell}^{\prime}} \cdot\left\langle u_{k}\right\rangle \frac{\partial \overline{u_{m}^{\prime}}}{\partial x_{k}}
$$

the term (iii) in Eq. (A8) can be expressed as follows:

$$
\begin{aligned}
& -\overline{u_{i}^{\prime}} \frac{\partial\left\langle u_{k}\right\rangle \overline{u_{j}^{\prime}}}{\partial x_{k}}-\overline{u_{j}^{\prime}} \frac{\partial\left\langle u_{k}\right\rangle \overline{u_{i}^{\prime}}}{\partial x_{k}}-\overline{u_{i}^{\prime}} \frac{\partial \overline{u_{k}^{\prime}}\left\langle u_{j}\right\rangle}{\partial x_{k}}-\overline{u_{j}^{\prime}} \frac{\partial \overline{u_{k}^{\prime}}\left\langle u_{i}\right\rangle}{\partial x_{k}} \\
& =-\overline{u_{i}^{\prime}} \cdot \overline{u_{k}^{\prime}} \frac{\partial\left\langle u_{j}\right\rangle}{\partial x_{k}}-\overline{u_{j}^{\prime}} \cdot \overline{u_{k}^{\prime}} \frac{\partial\left\langle u_{i}\right\rangle}{\partial x_{k}}-\overline{u_{i}^{\prime}} \cdot\left\langle u_{k}\right\rangle \frac{\partial \overline{u_{j}^{\prime}}}{\partial x_{k}}-\overline{u_{j}^{\prime}} \cdot\left\langle u_{k}\right\rangle \frac{\partial \overline{u_{i}^{\prime}}}{\partial x_{k}} \\
& =-\overline{u_{i}^{\prime}} \cdot \overline{u_{k}^{\prime}} \frac{\partial\left\langle u_{j}\right\rangle}{\partial x_{k}}-\overline{u_{j}^{\prime}} \cdot \overline{u_{k}^{\prime}} \frac{\partial\left\langle u_{i}\right\rangle}{\partial x_{k}}-\left\langle u_{k}\right\rangle\left(\overline{u_{i}^{\prime}} \frac{\partial \overline{u_{j}^{\prime}}}{\partial x_{k}}+\overline{u_{j}^{\prime}} \frac{\partial \overline{u_{i}^{\prime}}}{\partial x_{k}}\right) \\
& =-\overline{u_{i}^{\prime}} \cdot \overline{u_{k}^{\prime}} \frac{\partial\left\langle u_{j}\right\rangle}{\partial x_{k}}-\overline{u_{j}^{\prime}} \cdot \overline{u_{k}^{\prime}} \frac{\partial\left\langle u_{i}\right\rangle}{\partial x_{k}}-\left\langle u_{k}\right\rangle \frac{\partial\left(\overline{u_{i}^{\prime}} \cdot \overline{u_{j}^{\prime}}\right)}{\partial x_{k}} .
\end{aligned}
$$

We obtain

$$
\begin{aligned}
\frac{\partial\left(\overline{u_{i}^{\prime}} \cdot \overline{u_{j}^{\prime}}\right)}{\partial t}+\frac{\partial\left(\overline{u_{i}^{\prime}} \cdot \overline{u_{j}^{\prime}} \cdot \overline{u_{k}^{\prime}}\right)}{\partial x_{k}} \\
=-\frac{2}{\operatorname{Re}_{\tau}}\left(\frac{\partial \overline{u_{j}^{\prime}}}{\partial x_{k}} \frac{\partial \overline{u_{i}^{\prime}}}{\partial x_{k}}\right)+\frac{1}{\operatorname{Re}_{\tau}} \frac{\partial^{2}\left(\overline{u_{i}^{\prime}} \cdot \overline{u_{j}^{\prime}}\right)}{\partial x_{k} \partial x_{k}}+\overline{u_{i}^{\prime}} \frac{\partial\left\langle u_{k}^{\prime} u_{j}^{\prime}\right\rangle}{\partial x_{k}}+\overline{u_{j}^{\prime}} \frac{\partial\left\langle u_{k}^{\prime} u_{i}^{\prime}\right\rangle}{\partial x_{k}}-\overline{u_{i}^{\prime}} \cdot \overline{u_{k}^{\prime}} \frac{\partial\left\langle u_{j}\right\rangle}{\partial x_{k}} \\
\quad-\overline{u_{j}^{\prime}} \cdot \overline{u_{k}^{\prime}} \frac{\partial\left\langle u_{i}\right\rangle}{\partial x_{k}}-\left\langle u_{k}\right\rangle \frac{\partial\left(\overline{u_{i}^{\prime}} \cdot \overline{u_{j}^{\prime}}\right)}{\partial x_{k}}-\overline{u_{i}^{\prime}} \frac{\partial \tau_{k j}^{R}}{\partial x_{k}}-\overline{u_{j}^{\prime}} \frac{\partial \tau_{k i}^{R}}{\partial x_{k}}-\overline{u_{i}^{\prime}} \frac{\partial \overline{p^{\prime}}}{\partial x_{j}}-\overline{u_{j}^{\prime}} \frac{\partial \overline{p^{\prime}}}{\partial x_{i}} .
\end{aligned}
$$


Averaging gives the Reynolds stress budget equation for the filtered velocity field

$$
\begin{aligned}
0= & -\underbrace{\left(\frac{\partial\left\langle\overline{u_{i}^{\prime}} \cdot \overline{u_{j}^{\prime}}\right\rangle}{\partial t}+\left\langle u_{k}\right\rangle \frac{\partial\left\langle\overline{u_{i}^{\prime}} \cdot \overline{u_{j}^{\prime}}\right\rangle}{\partial x_{k}}\right)}_{\bar{D}^{*} / \bar{D} t}-\underbrace{\frac{\partial\left\langle\overline{u_{k}^{\prime}} \cdot \overline{u_{i}^{\prime}} \cdot \overline{u_{j}^{\prime}}\right\rangle}{\partial x_{k}}}_{T D_{i j}^{*}}+\underbrace{\frac{1}{\operatorname{Re}_{\tau}} \frac{\partial^{2}\left\langle\overline{u_{i}^{\prime}} \cdot \overline{u_{j}^{\prime}}\right\rangle}{\partial x_{k} \partial x_{k}}-\left\langle\overline{u_{i}^{\prime}} \frac{\partial \overline{p^{\prime}}}{\partial x_{j}}+\overline{u_{j}^{\prime}} \frac{\partial \overline{p^{\prime}}}{\partial x_{i}}\right\rangle}_{V D_{i j}^{*}} \underbrace{}_{\Pi_{i j}^{*}} \\
& \underbrace{-\left\langle\overline{u_{i}^{\prime}} \cdot \overline{u_{k}^{\prime}}\right\rangle \frac{\partial\left\langle u_{j}\right\rangle}{\partial x_{k}}-\left\langle\overline{u_{j}^{\prime}} \cdot \overline{u_{k}^{\prime}}\right\rangle \frac{\partial\left\langle u_{i}\right\rangle}{\partial x_{k}}}_{\mathcal{P}_{i j}^{*}}-\underbrace{\frac{2}{\operatorname{Re}_{\tau}}\left\langle\frac{\partial \overline{u_{j}^{\prime}}}{\partial x_{k}} \frac{\partial \overline{u_{i}^{\prime}}}{\partial x_{k}}\right\rangle}_{\epsilon_{i j}^{*}}-\left\langle\overline{u_{i}^{\prime}} \frac{\partial \tau_{k j}^{R}}{\partial x_{k}}\right\rangle-\left\langle\overline{u_{j}^{\prime}} \frac{\partial \tau_{k i}^{R}}{\partial x_{k}}\right\rangle .
\end{aligned}
$$

In comparison with the transport equation for the Reynolds stresses in Pope [24], p. 315, Eq. (7.178),

$$
\begin{aligned}
0= & -\left(\frac{\partial\left\langle u_{i}^{\prime} u_{j}^{\prime}\right\rangle}{\partial t}+\left\langle u_{k}\right\rangle \frac{\partial\left\langle u_{i}^{\prime} u_{j}^{\prime}\right\rangle}{\partial x_{k}}\right)-\frac{\partial\left\langle u_{k}^{\prime} u_{i}^{\prime} u_{j}^{\prime}\right\rangle}{\partial x_{k}}+\frac{1}{\operatorname{Re}_{\tau}} \frac{\partial^{2}\left\langle u_{i}^{\prime} u_{j}^{\prime}\right\rangle}{\partial x_{k} \partial x_{k}}-\left\langle u_{i}^{\prime} \frac{\partial p^{\prime}}{\partial x_{j}}+u_{j}^{\prime} \frac{\partial p^{\prime}}{\partial x_{i}}\right\rangle \\
& -\left\langle u_{i}^{\prime} u_{k}^{\prime}\right\rangle \frac{\partial\left\langle u_{j}\right\rangle}{\partial x_{k}}-\left\langle u_{j}^{\prime} u_{k}^{\prime}\right\rangle \frac{\partial\left\langle u_{i}\right\rangle}{\partial x_{k}}-\frac{2}{\operatorname{Re}_{\tau}}\left\langle\frac{\partial u_{j}^{\prime}}{\partial x_{k}} \frac{\partial u_{i}^{\prime}}{\partial x_{k}}\right\rangle,
\end{aligned}
$$

an additional term occurs due to spatial filtering. The additional term can be split into a transport term and a source/sink term for the residual stress

$$
\begin{aligned}
\left\langle\overline{u_{i}^{\prime}} \frac{\partial \tau_{k j}^{R}}{\partial x_{k}}+\overline{u_{j}^{\prime}} \frac{\partial \tau_{k i}^{R}}{\partial x_{k}}\right\rangle & =\left\langle\frac{\partial\left(\overline{u_{i}^{\prime}} \tau_{k j}^{R}\right)}{\partial x_{k}}-\tau_{k j}^{R} \frac{\partial \overline{u_{i}^{\prime}}}{\partial x_{k}}+\frac{\partial\left(\overline{u_{j}^{\prime}} \tau_{k i}^{R}\right)}{\partial x_{k}}-\tau_{k i}^{R} \frac{\partial \overline{u_{j}^{\prime}}}{\partial x_{k}}\right\rangle \\
& =\left(\frac{\partial\left\langle\overline{u_{i}^{\prime}} \tau_{k j}^{R}\right\rangle}{\partial x_{k}}+\frac{\partial\left\langle\overline{u_{j}^{\prime}} \tau_{k i}^{R}\right\rangle}{\partial x_{k}}\right)-\left\langle\tau_{k j}^{R} \frac{\partial \overline{u_{i}^{\prime}}}{\partial x_{k}}+\tau_{k i}^{R} \frac{\partial \overline{u_{j}^{\prime}}}{\partial x_{k}}\right\rangle .
\end{aligned}
$$

Substituting Eq. (A17) back into Eq. (A15) and evaluating the equation for the streamwise Reynolds stress component gives for fully developed turbulent pipe flow Eq. (6).

\section{APPENDIX B: ADDITIONAL FIGURES}

Additional figures are provided here. Figure 14 shows a comparison between the TKE interscale flux computed from the current study and the data from Härtel et al. [6]. Figures 15 and 16 present

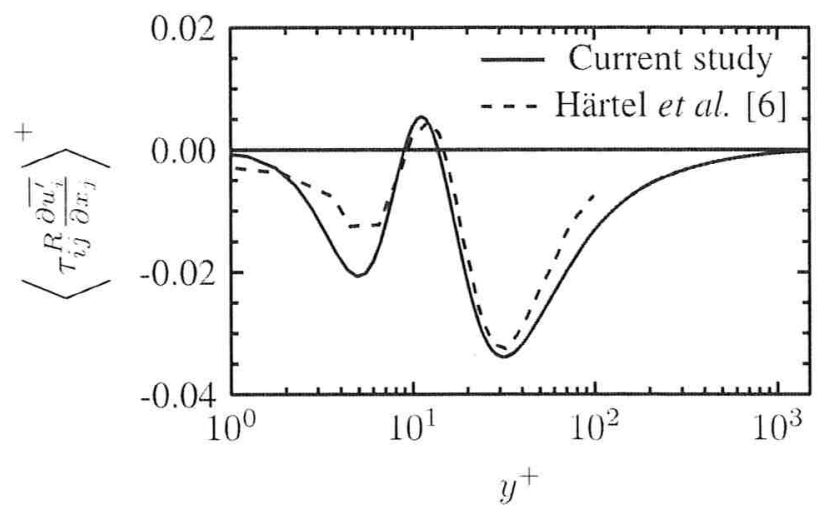

FIG. 14. Interscale energy in terms of turbulent kinetic energy $\left\langle\tau_{i j}^{R} \partial \overline{u_{i}^{\prime}} / \partial x_{j}\right\rangle^{+}$compared between Härtel et al. [6] $\left(\operatorname{Re}_{\tau}=180\right)$ and the current investigation investigation $\left(\operatorname{Re}_{\tau}=1500\right)$. Filter configuration $\mathrm{A}$. 


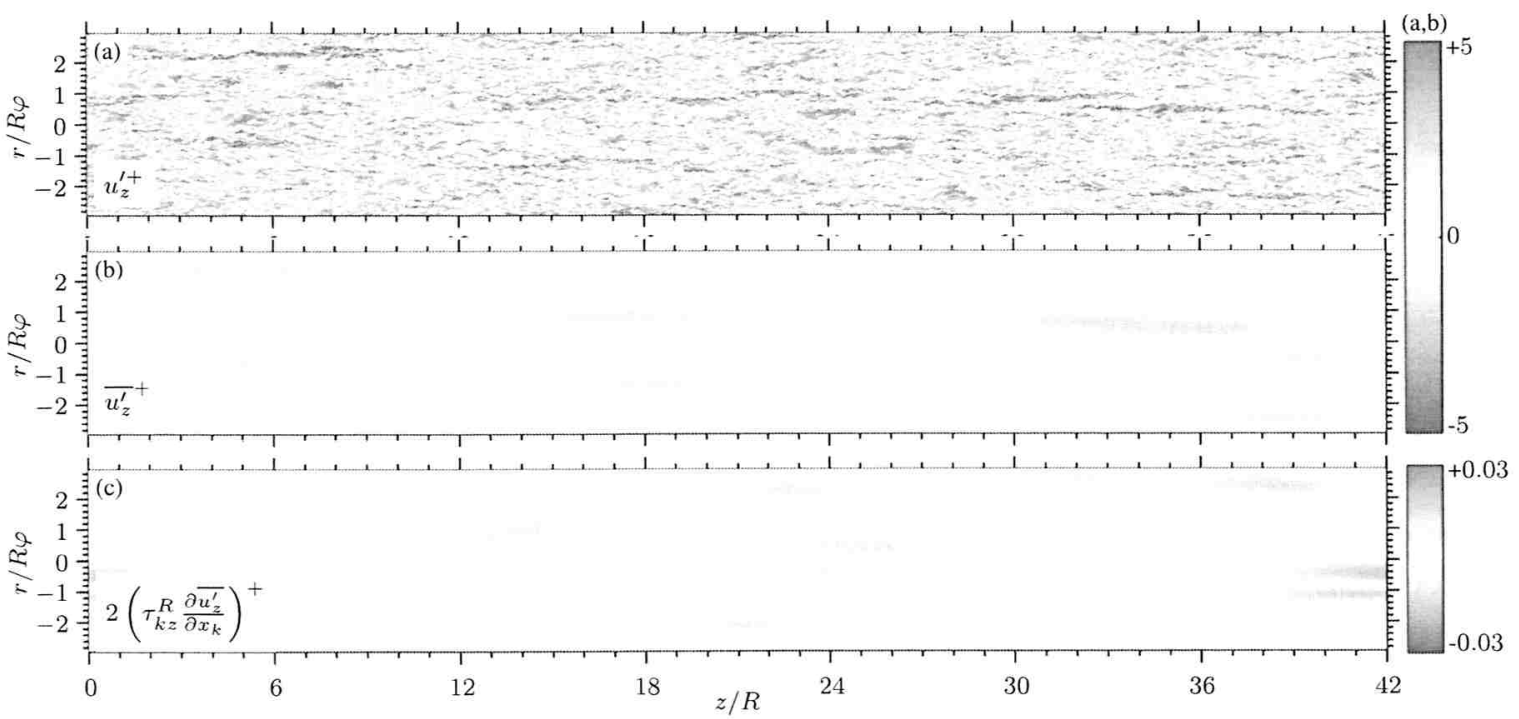

FIG. 15. Isocontours in a wall-parallel plane at $y^{+}=100$. (a) Unfiltered streamwise velocity fluctuation $u_{z}^{\prime}$. (b) Low-pass filtered streamwise velocity fluctuation $\overline{u_{z}^{\prime}}$. (c) Instantaneous interscale energy flux term, $2\left(\tau_{k z}^{R} \partial \overline{u_{z}^{\prime}} / \partial x_{k}\right)^{+}$. All terms normalized in wall units. Filter lengths: $\lambda_{z}^{+}=9000, \lambda_{\varphi}^{+}=1000$.

the unfiltered streamwise velocity field, the filtered streamwise velocity field (Configuration B), and the instantaneous interscale energy flux at two additional distances from the wall. The planes in Fig. 15 are located at $y^{+}=100$ and the ones in Fig. 16 at $y^{+}=300$.

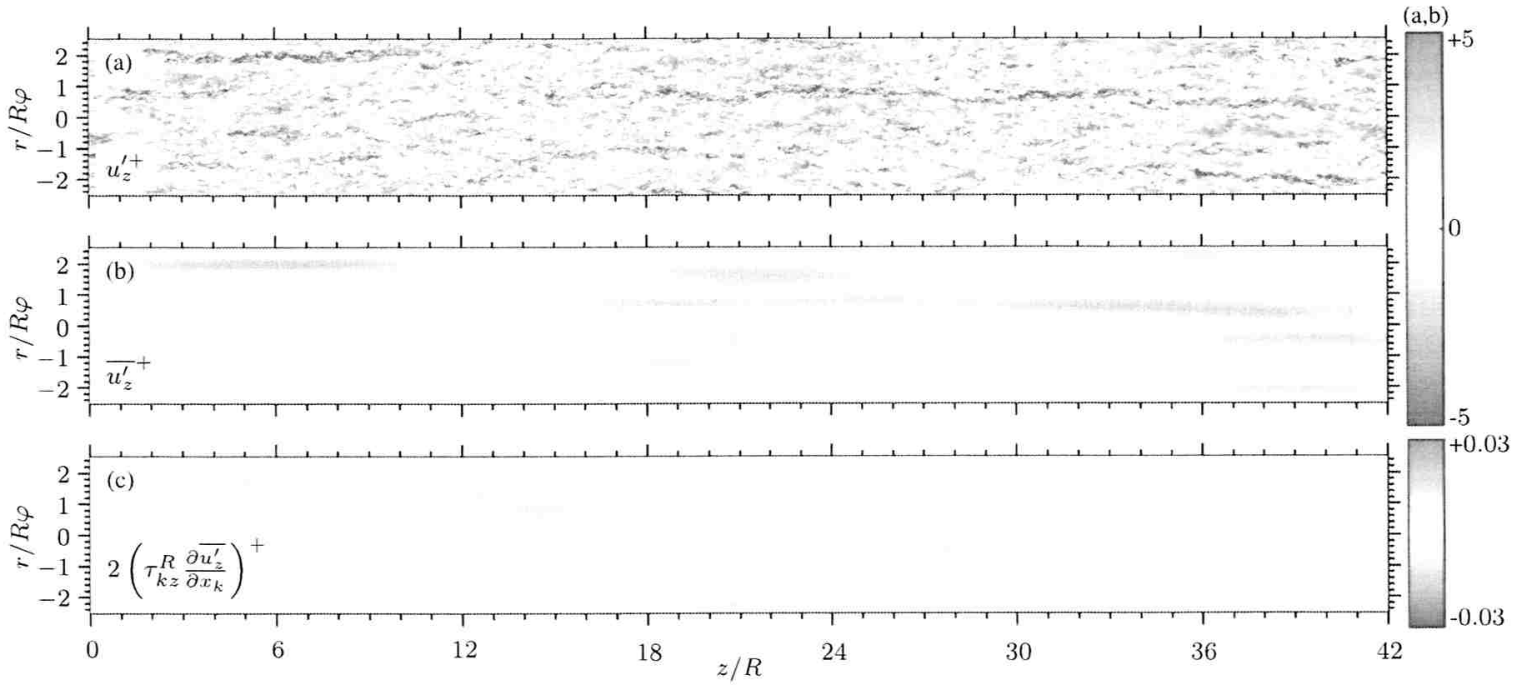

FIG. 16. Isocontours in a wall-parallel plane at $y^{+}=300$. (a) Unfiltered streamwise velocity fluctuation $u_{z}^{\prime}$. (b) Low-pass filtered streamwise velocity fluctuation $\overline{u_{z}^{\prime}}$. (c) Instantaneous interscale energy flux term, $2\left(\tau_{k z}^{R} \partial \overline{u_{z}^{\prime}} / \partial x_{k}\right)^{+}$. All terms normalized in wall units. Filter lengths: $\lambda_{z}^{+}=9000, \lambda_{\varphi}^{+}=1000$. 
[1] K. C. Kim and R. J. Adrian, Very large-scale motion in the outer layer, Phys. Fluids 11, 417 (1999).

[2] A. J. Smits, B. J. McKeon, and I. Marusic, High-Reynolds number wall turbulence, Annu. Rev. Fluid Mech. 43, 353 (2011).

[3] J. C. del Álamo and J. Jiménez, Spectra of the very large anisotropic scales in turbulent channels, Phys. Fluids 15, L41 (2003).

[4] C. Bauer, D. Feldmann, and C. Wagner, On the convergence and scaling of high-order statistical moments in turbulent pipe flow using direct numerical simulations, Phys. Fluids 29, 125105 (2017).

[5] S. Discetti, G. Bellani, R. Örlü, J. Serpieri, C. S. Vila, M. Raiola, X. Zheng, L. Mascotelli, A. Talamelli, and A. Ianiro, Characterization of very-large-scale motions in high-Re pipe flows, Experimental Thermal and Fluid Science 104, 1 (2019).

[6] C. Härtel, L. Kleiser, F. Unger, and R. Friedrich, Subgrid-scale energy transfer in the near-wall region of turbulent flows, Phys. Fluids 6, 3130 (1994).

[7] U. Piomelli, Y. Yu, and R. J. Adrian, Subgrid-scale energy transfer and near-wall turbulence structure, Phys. Fluids 8, 215 (1996).

[8] A. von Kameke, F. Huhn, G. Fernández-Garćia, A. P. Muñuzuri, and V. Pérez-Muñuzuri, Double Cascade Turbulence and Richardson Dispersion in a Horizontal Fluid Flow Induced by Faraday Waves, Phys. Rev. Lett. 107, 074502 (2011).

[9] T. Pestana and S. Hickel, Regime transition in the energy cascade of rotating turbulence, Phys. Rev. E 99, 053103 (2019).

[10] A. Cimarelli and E. De Angelis, Analysis of the Kolmogorov equation for filtered wall-turbulent flows, J. Fluid Mech. 676, 376 (2011).

[11] A. Cimarelli and E. De Angelis, Anisotropic dynamics and sub-grid energy transfer in wall-turbulence, Phys. Fluids 24, 015102 (2012).

[12] A. Cimarelli, E. De Angelis, and C. M. Casciola, Paths of energy in turbulent channel flows, J. Fluid Mech. 715, 436 (2013).

[13] A. Cimarelli and E. De Angelis, The physics of energy transfer toward improved subgrid-scale models, Phys. Fluids 26, 055103 (2014).

[14] A. Cimarelli, E. De Angelis, P. Schlatter, G. Brethouwer, A. Talamelli, and C. M. Casciola, Sources and fluxes of scale energy in the overlap layer of wall turbulence, J. Fluid Mech. 771, 407 (2015).

[15] A. Cimarelli, E. De Angelis, J. Jiménez, and C. M. Casciola, Cascades and wall-normal fluxes in turbulent channel flows, J. Fluid Mech. 796, 417 (2016).

[16] M. Cho, Y. Hwang, and H. Choi, Scale interactions and spectral energy transfer in turbulent channel flow, J. Fluid Mech. 854, 474 (2018).

[17] M. Lee and R. D. Moser, Spectral analysis of the budget equation in turbulent channel flows at high Reynolds number, J. Fluid Mech. 860, 886 (2019).

[18] C. Chin, A. S. H. Ooi, I. Marusic, and H. M. Blackburn, The influence of pipe length on turbulence statistics computed from direct numerical simulation data, Phys. Fluids 22, 115107 (2010).

[19] A. Lozano-Durán and J. Jiménez, Effect of the computational domain on direct simulations of turbulent channels up to $\operatorname{Re}_{T}=4200$, Phys. Fluids 26, 011702 (2014).

[20] D. Feldmann, C. Bauer, and C. Wagner, Computational domain length and Reynolds number effects on large-scale coherent motions in turbulent pipe flow, J. Turbulence 19, 274 (2018).

[21] O. Shishkina and C. Wagner, A fourth order finite volume scheme for turbulent flow simulations in cylindrical domains, Comput. Fluids 36, 484 (2007).

[22] T. Kawata and P. H. Alfredsson, Inverse Interscale Transport of the Reynolds Shear Stress in Plane Couette Turbulence, Phys. Rev. Lett. 120, 244501 (2018).

[23] M. Hultmark, M. Vallikivi, S. C. C. Bailey, and A. J. Smits, Turbulent Pipe Flow at Extreme Reynolds Numbers, Phys. Rev. Lett. 108, 094501 (2012).

[24] S. B. Pope, Turbulent Flows (Cambridge University Press, Cambridge, 2000).

[25] R. Mathis, N. Hutchins, and I. Marusic, Large-scale amplitude modulation of the small-scale structures in turbulent boundary layers, J. Fluid Mech. 628, 311 (2009). 
[26] R. Mathis, I. Marusic, N. Hutchins, and K. R. Sreenivasan, The relationship between the velocity skewness and the amplitude modulation of the small scale by the large scale in turbulent boundary layers, Phys. Fluids 23, 121702 (2011).

[27] M. Lee and R. D. Moser, Direct numerical simulation of turbulent channel flow up to $\operatorname{Re} \tau=5200$, J. Fluid Mech. 774, 395 (2015).

[28] J. Ahn, J. H. Lee, J. Lee, J.-h. Kang, and H. J. Sung, Direct numerical simulation of a 30R long turbulent pipe flow at $\operatorname{Re} \tau=3008$, Phys. Fluids 27, 065110 (2015).

[29] J. Ahn, J. Lee, and H. J. Sung, Contribution of large-scale motions to the Reynolds shear stress in turbulent pipe flows, Int. J. Heat Fluid Flow 66, 209 (2017).

[30] N. Marati, C. M. Casciola, and R. Piva, Energy cascade and spatial fluxes in wall turbulence, J. Fluid Mech. 521, 191 (2004).

[31] N. Saikrishnan, E. De Angelis, E. Longmire, I. Marusic, C. Casciola, and R. Piva, Reynolds number effects on scale energy balance in wall turbulence, Phys. Fluids 24, 015101 (2012).

[32] G. L. Eyink, Locality of turbulent cascades, Physica D: Nonlin. Phenom. 207, 91 (2005).

[33] J. M. Wallace, Quadrant analysis in turbulence research: History and evolution, Annu. Rev. Fluid Mech. 48, 131 (2016).

[34] R. J. Adrian, Hairpin vortex organization in wall turbulence, Phys. Fluids 19, 041301 (2007).

[35] Y. Hwang and C. Cossu, Self-Sustained Process at Large Scales in Turbulent Channel Flow, Phys. Rev. Lett. 105, 044505 (2010).

[36] Y. Hwang and C. Cossu, Self-sustained processes in the logarithmic layer of turbulent channel flows, Phys. Fluids 23, 061702 (2011).

[37] Y. Hwang, Statistical structure of self-sustaining attached eddies in turbulent channel flow, J. Fluid Mech. 767, 254 (2015).

[38] D. Feldmann and M. Avila, Overdamped large-eddy simulations of turbulent pipe flow up to $\operatorname{Re}_{T}=1500$, J. Phys.: Conf. Ser. 1001, 012016 (2018). 